\title{
ON THE DEFINING EQUATIONS OF ABELIAN VARIETIES
}

\section{HISASI MORIKAWA}

Dedicated to Professor Noshiro on the occation of his 60th birthday

This article is a continuation and completion of our study in the previous one [1] in which we gave the defining equations of abelian varieties projectively embedded by theta functions of level three. These defining equations are nothing else the canonical generators of theta relations for theta functions with $\frac{1}{3}$-characteristics. In the present article the canonical generators of theta relations will be given for the wider class of theta functions with rational characteristics.

Let $G$ be a finite subgroup in $\boldsymbol{Q}^{r} \mid \boldsymbol{Z}^{r}$ such that the index $\left[G: \frac{1}{3} \boldsymbol{Z}^{r} \mid \boldsymbol{Z}^{r}\right]$ is odd and $H^{+}$be the subset in $\frac{1}{3} \boldsymbol{Z}^{r} / \boldsymbol{Z}^{r}$ consisting of all the elements $\left(\left[\frac{a_{1}^{+}}{3}\right], \ldots .\right.$. , $\left.\left[\frac{a_{r}^{+}}{3}\right]\right)\left(a_{i}^{+}=0,1 ; i=1,2, \ldots \ldots, r\right)$. Then the canonical generators of theta relations for theta functions

$$
\vartheta_{[\boldsymbol{a}]}(Q \mid U)=\sum_{m \in Z^{r}} Q(\boldsymbol{m}+\boldsymbol{a}, \boldsymbol{m}+\boldsymbol{a}) U(\boldsymbol{m}+\boldsymbol{a})^{2} \quad([\boldsymbol{a}] \in G)
$$

are the following cubic and quadratic equations:

$(*)$

$$
\begin{aligned}
& \vartheta_{\left[\boldsymbol{a}^{\prime}\right]+[\boldsymbol{b}]}(Q \mid U) \vartheta_{-\left[\boldsymbol{a}^{\prime}\right]+[\boldsymbol{b}]}(Q \mid U) \vartheta_{[\boldsymbol{b}]}(Q \mid U) \\
& =\sum_{\left[\boldsymbol{c}^{\prime}\right] \in \frac{1}{3} \boldsymbol{Z}^{r} / \mathbf{Z}^{r}} \gamma_{\left[\boldsymbol{a}^{\prime}\right],\left[\boldsymbol{c}^{\prime}\right]}(Q) \vartheta_{\left[\boldsymbol{c}^{\prime}\right]+[\boldsymbol{b}]}(Q \mid U)^{3}=0 \\
& \vartheta_{[\boldsymbol{a}]+[b]}(Q \mid U) \vartheta-[\boldsymbol{a}]+[\boldsymbol{b}](Q \mid U) \\
& =\sum_{\left[\boldsymbol{c}^{+}\right] \in H^{+}} \beta_{[\boldsymbol{a}],\left[\boldsymbol{c}^{+}\right]}(Q) \vartheta_{\left[\boldsymbol{c}^{+}\right]+[\boldsymbol{b}]}(Q \mid U) \vartheta_{-\left[\boldsymbol{c}^{+}\right]+[\boldsymbol{b}]}(Q \mid U)=0 \\
& \left(\left[\boldsymbol{a}^{\prime}\right] \in \frac{1}{3} \boldsymbol{Z}^{r} / \boldsymbol{Z}^{r} ;[\boldsymbol{a}],[\boldsymbol{b}] \in G\right)
\end{aligned}
$$

where the coefficients $\gamma_{\left[\boldsymbol{a}^{\prime}\right],\left[\boldsymbol{c}^{\prime}\right]}(Q)\left(\left[\boldsymbol{a}^{\prime}\right],\left[\boldsymbol{c}^{\prime}\right] \in \frac{1}{3} \boldsymbol{Z}^{r} / \boldsymbol{Z}^{r}\right)$, in the cubic equations do not depend on $G$.

Received August 15, 1966. 


\section{TERMINOLOGY AND NOTATIONS}

$\boldsymbol{Q}^{r}$ : the coordinate vector space of dimension $r$ over the field of rational numbers,

$\boldsymbol{Z}^{r}$ : the subgroup of $\boldsymbol{Q}^{r}$ consisting of all the integral vectors, i.e. vectors with integral coordinates,

$\boldsymbol{Q}^{r} / \boldsymbol{Z}^{r}$ : the residue group of $\boldsymbol{Q}^{r}$ by $\boldsymbol{Z}^{r}$,

$[\boldsymbol{a}]$ : the class of a vector $\boldsymbol{a}$ in the residue group $\boldsymbol{Q}^{r} / \boldsymbol{Z}^{r}$

[a] : the class of a rational number $\boldsymbol{a}$ in $\boldsymbol{Q} / \boldsymbol{Z}$,

$H$ : the subgroup $\frac{1}{3} \boldsymbol{Z}^{r} \mid \boldsymbol{Z}^{r}$ in $\boldsymbol{Q}^{r} \mid \boldsymbol{Z}^{r}$, i.e. $H=\left\{\left(\left[\frac{a_{1}}{3}\right], \ldots \ldots,\left[\frac{a_{r}}{3}\right]\right)\right.$ $\left.a_{i} \in\{0,1,-1\}(1 \leq i \leq r)\right\}$,

$H^{+}$: the subset in $H$ consisting of all the elements $\left(\left[\frac{a_{1}}{3}\right], \ldots . .,\left[\frac{a_{r}}{3}\right]\right)$ such that $a_{i} \in\{0,1\}(1 \leq i \leq r)$

$\left[\boldsymbol{a}^{\prime}\right],\left[\boldsymbol{b}^{\prime}\right],\left[\boldsymbol{c}^{\prime}\right], \ldots . .:$ : the elements in $H$, $\left[\boldsymbol{a}^{+}\right],\left[\boldsymbol{b}^{+}\right],\left[\boldsymbol{c}^{+}\right], \ldots \ldots$ : the elements in $H^{+}$,

$G$ : a finite subgroup of $\boldsymbol{Q}^{r} / \boldsymbol{Z}^{r}$ such that $G \supset H$,

$|G|$ : the order of $G$,

$X_{[\boldsymbol{a}]}\left([\boldsymbol{a}] \in \boldsymbol{Q}^{r} / \boldsymbol{Z}^{r}\right):$ a system of indeterminates,

$T_{[\boldsymbol{a}]}\left([\boldsymbol{a}] \in \boldsymbol{Q}^{r} / \boldsymbol{Z}^{r}\right):$ a fixed system of generic solutions for

$$
X_{[\boldsymbol{a}]}-X_{-[\boldsymbol{a}]}=0 \quad\left([\boldsymbol{a}] \in \boldsymbol{Q}^{r} / \boldsymbol{Z}^{r}\right),
$$

$\left(T_{\left[a^{+}\right]+\left[b^{+}\right]} T-\left[a^{+}\right]+\left[b^{+}\right]\right)_{H^{+} \times H^{+}}:$the $2^{r} \times 2^{r}$ martrix of which $\left(\left[\boldsymbol{a}^{+}\right],\left[\boldsymbol{b}^{+}\right]\right)$-component is $T_{\left[a^{+}\right]+\left[b^{+}\right]} T_{-\left[a^{+}\right]+\left[b^{+}\right]}\left(\left[\boldsymbol{a}^{+}\right],\left[\boldsymbol{b}_{+}\right] \in H^{+}\right)$,

$\left(T_{[a]+\left[b^{+}\right]} T_{-[a]+\left[b^{+}\right]}\right)_{G \times H}$ : the $|G| \times 2^{r}$-matrix of which $\left([\boldsymbol{a}],\left[\boldsymbol{b}^{+}\right]\right)$-componen is $T_{[\boldsymbol{a}]+\left[\boldsymbol{b}^{+}\right]} T_{-[\boldsymbol{a}]+\left[\boldsymbol{b}^{+}\right]}\left([\boldsymbol{a}] \in G,\left[\boldsymbol{b}^{+}\right] \in H^{+}\right)$,

$\left(T_{[a]+\left[c^{+}\right]+\left[d^{+}\right]} T_{[a]-\left[c^{+}\right]+\left[d^{+}\right]}\right)_{H^{+} \times H^{+}}:$the $2^{r} \times 2^{r}$-matrix of which $\left(\left[c^{+}\right],\left[d^{+}\right]\right)$-component

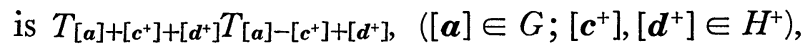

$\left(T_{\left[a^{\prime}\right]+\left[b_{1}\right]} T_{-\left[a^{\prime}\right]+\left[b^{\prime}\right]} T_{\left[b^{\prime}\right]}\right)_{H \times H}$ : the $3^{r} \times 3^{r}$-martix of which $\left(\left[\boldsymbol{a}^{\prime}\right],\left[\boldsymbol{b}^{\prime}\right]\right)$-component is $T_{\left[a^{\prime}\right]+\left[b^{\prime}\right]} T-\left[a^{\prime}\right]+\left[b^{\prime}\right] T_{\left[b^{\prime}\right]}\left(\left[\boldsymbol{a}^{\prime}\right],\left[\boldsymbol{b}^{\prime}\right] \in H\right)$,

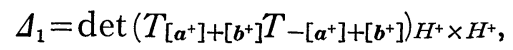

$\Delta_{2}=\operatorname{det}\left(T^{3}\left[a^{\prime}\right]+\left[b^{\prime}\right]\right)_{H \times H}$,

$\left(\alpha_{\left[a^{+}\right],\left[b^{+}\right]}\right)_{H^{+} \times H^{+}}:$the adojoint matrix of $\left(T_{\left[a^{+}\right]+\left[b^{+}\right]} T_{\left.\left.-\left[a^{+}\right]+\right] b^{+}\right]}\right)_{H^{+} \times H^{+}}$,

$\left(\gamma_{\left[a^{\prime}\right],\left[b^{\prime}\right]}\right)_{H \times H}=\left(T_{\left[a^{\prime}\right]+\left[b^{\prime}\right]} T-\left[a^{\prime}\right]+\left[b^{\prime}\right] T_{\left[b^{\prime}\right]}\right)_{H \times H}\left(T^{3}\left[a^{\prime}\right]+\left[b^{\prime}\right]\right)_{H \times H}^{-1}$

$\left(\beta[a],\left[b^{+}\right]\right)_{H^{+} \times H^{+}}=\left(T_{[a]+\left[b^{+}\right]} T-[a]+\left[b^{+}\right]\right) G \times H^{+}\left(T_{\left[a^{+}\right]+\left[b^{+}\right]} T_{-\left[a^{+}\right]+\left[b^{+}\right]}\right)_{H^{+} \times H^{+}}^{-1}$

$\S 1$ Formal theta functinos with rational characteristics.

1.1 We mean by $\{W(i ; a), W(j, ; b) \mid 1<i, j \leq r ; a, b \in \boldsymbol{Q}\}$ a system of indeterminates on which rational numbers operates as follows: 


$$
W(i ; a)^{c}=W(i ; a c), W(j, l ; b)^{c}=W(j, l ; b c)(1 \leq i, j \leq r ; a, b, c \in \mathbf{Q}) .
$$

Let $\mathbf{I}$ be the ideal in the polynomial ring $Z[\{W(i ; a), W(j, l ; b)\}]$ generated by

$$
\begin{aligned}
& W(i ; 0)-1, W(j, l ; 0)-1, W(j ; n a)-W(j ; a)^{n}, W(j, l ; n b)-W(j, l ; b)^{n}, \\
& W(i ; a) W(i ; b)-W(i ; a+b), W(j, l ; a) W(j, l ; b)-W(j, l ; a+b), \\
& W(j, l ; b)-W(l, j ; b)
\end{aligned}
$$$$
(1 \leq i, j, l \leq r ; a, b \in \boldsymbol{Q} ; n=1,2,3, \ldots \ldots)
$$

Denote by $U_{i}^{a}$ and $Q_{j}^{b}$ the images of $W(i ; a)$ and $W(j, l ; b)$ in the resdue ring $B=Z[\{W(i ; a), W(j, l ; b)\}] / \boldsymbol{I}$, respectively. Then it follows

$$
\begin{gathered}
U_{i}^{0}=1, Q_{j},=1, \quad U_{i}^{n a}=\overbrace{U_{i}^{a} \ldots \ldots U_{i}^{a}}^{n}, Q_{j,}^{n b}=\overbrace{Q_{j}^{b} \ldots \ldots Q_{j}^{b},}^{n}, U_{i}^{a} U_{i}^{b}=U_{i}^{a+b}, Q_{j, l}^{a} Q_{j, l}^{b}=Q_{j, l}^{a+b} \\
(1 \leq i, j, l \leq r ; a, b \in \boldsymbol{Q} ; n=1,2,3, \ldots \ldots)
\end{gathered}
$$

Using the briel notations

$$
\begin{aligned}
U(\boldsymbol{a})=\prod_{i=1}^{r} U_{i}^{a_{i}}, & Q(a, b)=\sum_{j, l=1}^{r} Q_{j, l}^{a_{j} b_{l}} \\
& \left(\boldsymbol{a}=\left(a_{2}, \ldots \ldots, a_{r}\right), \quad \boldsymbol{b}=\left(b_{1}, \ldots \ldots, b_{r}\right)\right)
\end{aligned}
$$

we have the following multiplicative rules:

$$
\begin{gathered}
U(\boldsymbol{a})^{c}=U(\boldsymbol{c a}), \quad Q(\boldsymbol{a}, \boldsymbol{b})^{c}=Q(c \boldsymbol{a}, \boldsymbol{b})=Q(\boldsymbol{a}, \boldsymbol{b}) \\
U(\boldsymbol{a}) U(\boldsymbol{b})=U(\boldsymbol{a}+\boldsymbol{b}), \quad Q(\boldsymbol{a}+\boldsymbol{b}, \boldsymbol{c}+\boldsymbol{d})=Q(\boldsymbol{a}, \boldsymbol{c}) Q(\boldsymbol{b}, \boldsymbol{c}) Q(\boldsymbol{a}, \boldsymbol{d}) Q(\boldsymbol{b}, \boldsymbol{d}) \\
\left(\boldsymbol{a}, \boldsymbol{b}, \boldsymbol{c}, \boldsymbol{d} \in \boldsymbol{Q}^{r} ; c \in \boldsymbol{Q}\right) .
\end{gathered}
$$

Denote by Hom $\left(\boldsymbol{Q}^{r} ; \boldsymbol{G}_{m}\right)(B)$ the group of all the homorphisms of $\boldsymbol{Q}^{r}$ into the multiplicative group of units in the ring $B$. Then $U$ and $Q(\boldsymbol{a})\left(\boldsymbol{a} \in \boldsymbol{Q}^{r}\right)$ may be regarded as the elements in Hom $\left(\boldsymbol{Q}^{r} ; G_{m}\right)(B)$ such that

$$
\begin{aligned}
& U: \boldsymbol{c} \\
& Q(\boldsymbol{a}): \boldsymbol{c} \rightarrow Q(\boldsymbol{c}) \\
& \rightarrow(\boldsymbol{a})(\boldsymbol{c})=Q(\boldsymbol{a}, \boldsymbol{c}) \quad\left(\boldsymbol{c} \in \boldsymbol{Q}^{r}\right) .
\end{aligned}
$$

The product $Q(\boldsymbol{a}) U$ and $Q(\boldsymbol{a}) Q(\boldsymbol{b})$ mean the elements in Hom $\left(\boldsymbol{Q}^{r}, \boldsymbol{G}_{m}\right)(B)$ defined by

$$
\begin{aligned}
& Q(\boldsymbol{a}) U: \boldsymbol{c} \rightarrow Q(\boldsymbol{a}, \boldsymbol{c}) U(\boldsymbol{c}) \\
& Q(\boldsymbol{a}) Q(\boldsymbol{b}): \boldsymbol{c} \rightarrow Q(\boldsymbol{a}, \boldsymbol{c}) Q(\boldsymbol{b}, \boldsymbol{c})=Q(\boldsymbol{a}+\boldsymbol{b}, \boldsymbol{c})\left(\boldsymbol{c} \in \boldsymbol{Q}^{r}\right),
\end{aligned}
$$

i.e. $Q(\boldsymbol{a}) Q(\boldsymbol{b})=Q(\boldsymbol{a}+\boldsymbol{b})$.

1.2 Similarly as the classical case first of all we shall introduce the theta 
functions with rational characteristics:

$$
\vartheta_{[a]}(Q \mid U)=\sum_{\boldsymbol{m} \in \boldsymbol{Z}^{r}} Q(\boldsymbol{m}+\boldsymbol{a}, \boldsymbol{m}+\boldsymbol{a}) U(\boldsymbol{m}+\boldsymbol{a})^{2} \quad\left([\boldsymbol{a}] \in \boldsymbol{Q}^{r} / \boldsymbol{Z}^{r}\right)
$$

The function $\vartheta_{[a]}(Q \mid U)$ does not depend on the choice of the representative $\boldsymbol{a}$ of $[\boldsymbol{a}]$. The theta zero values are also defined by

$$
\vartheta_{[a]}(Q)=\sum_{\boldsymbol{m} \in \boldsymbol{Z}^{r}} Q(\boldsymbol{m}+\boldsymbol{a}, \boldsymbol{m}+\boldsymbol{a}) \quad\left([\boldsymbol{a}] \in \boldsymbol{Q}^{r} / \boldsymbol{Z}^{r}\right) .
$$

The theta zero-value $\vartheta_{[a]}(Q)$ is regarded as the specialization of $\vartheta_{[a]}(Q \mid U)$ with respect to the replacement of $U(\boldsymbol{b})\left(\boldsymbol{b} \in \boldsymbol{Q}^{r}\right)$ by 1 .

From the definitions (1) and (2) it follows the following formulae:

$$
\begin{gathered}
\vartheta_{[\boldsymbol{a}]}(Q \mid Q(\boldsymbol{l}) U)=Q(\boldsymbol{l}, \boldsymbol{l})^{-1} U(\boldsymbol{l})^{-2} \vartheta_{[\boldsymbol{a}]}(Q \mid U) \quad\left(\boldsymbol{l} \in \boldsymbol{Z}^{r}\right) \\
\vartheta_{[\boldsymbol{a}]}\left(Q \mid U^{-1}\right)=\vartheta_{-[\boldsymbol{a}]}(Q \mid U) \\
\vartheta_{[\boldsymbol{a}]}(Q \mid Q(\boldsymbol{b}) U)=Q(\boldsymbol{b}, \boldsymbol{b})^{-1} U(\boldsymbol{b})^{-2} \vartheta_{[\boldsymbol{a}+\boldsymbol{b}]}(Q \mid U) \\
\vartheta_{[\boldsymbol{a}]}(Q)=\vartheta_{-[\boldsymbol{a}]}(Q) \\
\vartheta_{[\boldsymbol{a}+\boldsymbol{b}]}(Q)=Q(\boldsymbol{b}, \boldsymbol{b}) \vartheta_{[\boldsymbol{a}]}(Q \mid Q(\boldsymbol{b})) .
\end{gathered}
$$

The products of $\vartheta_{[\boldsymbol{a}]}(Q \mid U), \vartheta_{[\boldsymbol{b}]}(Q)\left([\boldsymbol{a}],[\boldsymbol{b}] \in \boldsymbol{Q}^{r} / \boldsymbol{Z}^{r}\right)$ can be defined as series in $U$ and $Q$, hence the polynomial ring $Z\left[\left\{\vartheta_{[a]}(Q \mid U), \vartheta_{[b]}(Q)\right\}\right]$ is well-defined.

For each subset in $\boldsymbol{Q}^{r} / \boldsymbol{Z}^{r}$ denote by $S^{*}$ the subset in $\boldsymbol{Q}^{r}$ such that $S^{*} \supset \boldsymbol{Z}^{r}$ and the image $S^{*} / \boldsymbol{Z}^{r}$ coincides with $S$. Let us give the abstract definition for formal theta functions.

Definition 1. Let $n$ be a positive integer and $S$ be a finite subset in $\boldsymbol{Q}^{r} \mid \boldsymbol{Z}^{r}$. Then a formal series $\varphi(U)=\sum_{\boldsymbol{m} \in S^{*}} \lambda_{\boldsymbol{m}} U(\boldsymbol{m})^{2}$ is called a formal theta function of type $(n ; S)$ with coefficients in a ring $A$ if $\lambda_{\boldsymbol{m}} Q(\boldsymbol{m}, \boldsymbol{m})^{\frac{-1}{n}}\left(\boldsymbol{m} \in S^{*}\right)$ belong to $A$ and $\varphi(U)$ satisfies the difference relation:

$$
\varphi(Q(\boldsymbol{l}) U)=Q(\boldsymbol{l}, \boldsymbol{l})^{-n} U(\boldsymbol{l})^{-2 n} \varphi(U) \quad\left(\boldsymbol{l} \in \boldsymbol{Z}^{r}\right) .
$$

We mean by $\boldsymbol{M}_{A}(n ; S)$ the $A$ module of formal theta functions of type $(n ; S)$.

Proposition 1. Let $n$ be a positive integer and $S$ be a finite subset in $\boldsymbol{Q}^{r} / \boldsymbol{Z}^{r}$. Then for any field $k$ it follows

$$
\operatorname{dim}_{k} \boldsymbol{M}_{k}(n ; S)=\left|\frac{1}{n} S\right|=n^{r}|S|
$$

where $\left|\frac{1}{n} S\right|$ be the cardinal of the subset $\{[\boldsymbol{b}] \mid n[\boldsymbol{b}] \in S\}$ and $|S|$ is the cardinal 
of $S$.

Proof. Let $b_{1}, \ldots \ldots, b_{n^{r}|S|}$ be a system of representatives of $\frac{1}{n} S$ in $\boldsymbol{Q}^{r}$ and $\sum_{\boldsymbol{m} \in S^{*}} \lambda_{m} U(\boldsymbol{m})^{2}$ be a formal theta function of type $(n ; S)$ with coefficients in $k$. Put

$$
\sum_{m \in S^{*}} \lambda_{m} U(\boldsymbol{m})^{2}=\sum_{i=1}^{n^{r}|S|} \sum_{\boldsymbol{l} \in \boldsymbol{Z}^{r}} \lambda_{n}\left(\boldsymbol{l}+\boldsymbol{b}_{i}\right) U\left(n\left(\boldsymbol{l}+\boldsymbol{b}_{i}\right)\right)^{2} .
$$

Then by virtue of the difference relation (8) it follows

$$
\begin{aligned}
& \sum_{\boldsymbol{l} \in \boldsymbol{Z}^{r}} \lambda_{n\left(\boldsymbol{l}+\boldsymbol{b}_{i}\right)} U\left(n\left(\boldsymbol{l}+\boldsymbol{b}_{i}\right)\right)^{2} Q\left(n\left(\boldsymbol{l}+\boldsymbol{b}_{i}\right), \boldsymbol{h}\right)^{2} \\
& \quad=Q(\boldsymbol{h}, \boldsymbol{h})^{-n} U(\boldsymbol{h})^{-2 n} \sum_{\boldsymbol{l} \in \boldsymbol{Z}^{\tau}} \lambda_{n\left(\boldsymbol{l}+\boldsymbol{b}_{i}\right)} U\left(n\left(\boldsymbol{l}+\boldsymbol{b}_{\imath}\right)\right)^{2}, \quad\left(\boldsymbol{h} \in \boldsymbol{Z}^{r}\right)
\end{aligned}
$$

Hence

$$
\begin{aligned}
& \sum_{\boldsymbol{l} \in \mathbf{Z}^{r}} \lambda n\left(\boldsymbol{l}+\boldsymbol{b}_{i}\right) Q(\boldsymbol{h}, \boldsymbol{h})^{n} Q\left(\boldsymbol{l}+\boldsymbol{b}_{i}, \boldsymbol{h}\right)^{2 n} U\left(\boldsymbol{l}+\boldsymbol{h}+\boldsymbol{b}_{i}\right)^{2 n} \\
& =\sum_{\boldsymbol{l} \in \boldsymbol{Z}^{r}} \lambda_{n\left(\boldsymbol{l}+\boldsymbol{h}+\boldsymbol{b}_{i}\right)} U\left(\boldsymbol{l}+\boldsymbol{h}+\boldsymbol{b}_{i}\right)^{2 n} \quad\left(\boldsymbol{l} \in \boldsymbol{Z}^{r}\right) .
\end{aligned}
$$

This means

$$
\begin{aligned}
& \lambda_{n\left(\boldsymbol{l}+\boldsymbol{b}_{i}\right)} Q(\boldsymbol{h}, \boldsymbol{h})^{n} Q\left(\boldsymbol{h}, \boldsymbol{l}+\boldsymbol{b}_{i}\right)^{2 n}=\lambda_{n\left(\boldsymbol{l}+\boldsymbol{h}+\boldsymbol{b}_{i}\right),} \\
& \lambda_{n \boldsymbol{b}_{i}} Q\left(\boldsymbol{b}_{\imath}, \boldsymbol{b}_{i}\right)^{-n} Q\left(\boldsymbol{h}+\boldsymbol{b}_{i}, \boldsymbol{h}+\boldsymbol{b}_{i}\right)^{n}=\lambda_{n\left(\boldsymbol{h}+\boldsymbol{b}_{i}\right)} \\
& \left(\boldsymbol{l}, \boldsymbol{h} \in \boldsymbol{Z}^{r} ; 1 \leq i \leq n^{r}|S|\right) .
\end{aligned}
$$

Therefore

$$
\begin{aligned}
\sum_{\boldsymbol{l} \in \boldsymbol{Z}^{r}} \lambda_{n} & \left.\boldsymbol{l} \boldsymbol{l}+\boldsymbol{b}_{i}\right) \\
& =\lambda_{n \boldsymbol{b}_{i}} Q\left(n\left(\boldsymbol{l}+\boldsymbol{b}_{i}, \boldsymbol{b}^{i}\right)^{-n} \sum_{\boldsymbol{l} \in \boldsymbol{Z}^{r}} Q\left(\boldsymbol{l}+\boldsymbol{b}_{i}, \boldsymbol{l}+\boldsymbol{b}_{i}\right)^{n} U\left(\boldsymbol{l}+\boldsymbol{b}_{i}\right)^{n}\right. \\
& =\lambda_{n \boldsymbol{b}_{i}} Q\left(\boldsymbol{b}_{i}, \boldsymbol{b}_{i}\right)^{-n} \vartheta_{\left[\boldsymbol{b}_{i}\right]}\left(Q^{n} \mid U^{n}\right) \\
& =\lambda_{n \boldsymbol{b}_{i}} Q\left(n \boldsymbol{b}_{i}, n \boldsymbol{b}_{i}\right)^{-\frac{1}{n}} \vartheta_{\left[\boldsymbol{b}_{i}\right]}\left(Q^{n} \mid U^{n}\right)
\end{aligned}
$$

This means that the partial sums $\sum_{\boldsymbol{l}=\boldsymbol{Z}^{r}} \lambda_{n\left(\boldsymbol{l}+\boldsymbol{b}_{i}\right)} U\left(n\left(\boldsymbol{l}+\boldsymbol{b}_{i}\right)\right)\left(1 \leq i \leq n^{r}|S|\right)$ belong to $\boldsymbol{M}_{k}(n ; S)$. Since $\vartheta_{\left[b_{i}\right]}\left(Q^{n} \mid U^{n}\right) \quad\left(1 \leq i \leq n^{r} s\right)$ have mutually different terms in $U$ and they are elements in $\boldsymbol{M}_{k}(n ; S)$, we conclude that $\vartheta_{\left[b_{i}\right]}\left(Q^{n} \mid U^{n}\right)\left(1 \leq i \leq n^{\gamma} s\right)$ form a base of $\boldsymbol{M}_{k}(n ; S)$. This proves Proposition 1 .

LeMma 1. For any prime number $p$

(9) $\operatorname{det}\left(\vartheta_{\left[\boldsymbol{a}^{+}\right]+\left[\boldsymbol{b}^{+}\right]}(Q) \vartheta_{-\left[\boldsymbol{a}^{+}\right]+\left[\boldsymbol{b}^{+}\right]}(Q)\right)_{H^{+} \times H^{+}} \neq \equiv \bmod p$, where $\left(\vartheta_{\left[\boldsymbol{a}^{+}\right]+\left[\boldsymbol{b}^{+}\right]}(Q) \vartheta_{-\left[\boldsymbol{a}^{+}\right]+\left[\boldsymbol{b}^{+}\right]}(Q)\right)_{H^{+} \times H^{+}}$is the $2^{r} \times 2^{r}$-matrix of which $\left[\boldsymbol{a}^{+}\right] \times\left[\boldsymbol{b}^{+}\right]$-element is $\vartheta_{\left[a^{+}\right]+\left[b^{+}\right]}(Q) \vartheta-\left[a^{+}\right]+\left[b^{+}\right](Q)$.

The proof of Lemma 1 will be given in the next paragraph. 
Proposition 2. Let $k$ be a field such that $\vartheta_{\left[c^{+}\right]+[a]}(Q \mid U) \vartheta_{\left[c^{+}\right]+[a]}(Q \mid U)\left(\left[c^{+}\right] \in H^{+}\right)$ belong to $\boldsymbol{M}_{k}(2 ; 2[\boldsymbol{a}])$. Then $\vartheta_{\left[\boldsymbol{c}^{+}\right]+[\boldsymbol{a}]}(Q \mid U) \vartheta_{-\left[\boldsymbol{c}^{+}\right]+[a]}(Q \mid U)\left(\left[\boldsymbol{c}^{+}\right] \in H^{+}\right)$form a base of $\boldsymbol{M}_{k}(2 ; 2[\boldsymbol{a}])$.

Proof. Since $\operatorname{dim}_{k} \boldsymbol{M}_{k}(2 ; 2[\boldsymbol{a}])=2^{r}$, it is sufficient to shows that $\vartheta_{\left[c^{+}\right]+[a]}(Q \mid U) \vartheta_{\left.-] c^{+}\right]+[a]}(Q \mid U)\left(\left[c^{+}\right] \in H^{+}\right)$are linearly independent over $k$. From (7) it follows

$$
\begin{aligned}
\vartheta_{\left[c^{+}\right]+\left[b^{+}\right]}(Q) & \vartheta_{-\left[c^{+}\right]+\left[b^{+}\right]}(Q) \\
& =Q\left(b^{+}-a, b^{+}-a\right)^{2} \vartheta_{\left[c^{+}+a\right]}\left(Q \mid Q\left(b^{+}-a\right) \vartheta_{\left[-c^{+}+a\right]}\left(Q \mid Q\left(b^{+}-a\right)\right)\right.
\end{aligned}
$$

This means that $\vartheta_{\left[c^{+}+b^{+}\right]}(Q) \vartheta_{-\left[c^{+}\right]+\left[b^{+}\right]}(Q)$ is the specialization of $Q\left(\boldsymbol{b}^{+}-\boldsymbol{a}, \boldsymbol{b}^{+}-\boldsymbol{a}\right)^{2} \vartheta_{\left[\boldsymbol{c}^{+}\right]+[\boldsymbol{a}]}(Q \mid U) \vartheta_{-\left[\boldsymbol{c}^{+}\right]+[\boldsymbol{a}]}(Q \mid U)$ induced by the replacement: $U \rightarrow Q\left(\boldsymbol{b}^{+}-\boldsymbol{a}\right)$. Hence the relation

$$
\operatorname{det} \quad\left(\vartheta_{\left.\left.\left[a^{+}\right]+\right] b^{+}\right]}(Q) \vartheta_{-\left[a^{+}\right]+\left[b^{+}\right]}(Q)\right)_{H^{+} \times H^{+}} \equiv \equiv \quad \bmod p
$$

implies that $\vartheta_{\left[c^{+}\right]+[a]}(Q \mid U) \vartheta_{-\left[c^{+}\right]+[a]}(Q \mid U) \quad\left(\left[c^{+}\right] \in H^{+}\right)$are linearly independent over $k$.

§2. Defining equations of canonical elliptic curves.

2.1 The most beautifly explicite expressions of plane cubics are Hessian canonical forms: $X_{0}^{3}+X_{1}^{3}+X_{2}^{3}-3 \gamma X_{0} X_{1} X_{2}=0$. If $\gamma^{3} \neq 1$, the Hesse's canonical cubics are non-singular and conversely, for the classical case, any non-singular plane cubic is projectively transformed to a Hesse's canonical cubic.

We shall first recall that the projective locus of the formal thetas $\vartheta_{[0]}(Q \mid U)$, $\left.\vartheta_{\left[\frac{1}{3}\right]}(Q \mid U), \vartheta_{\left[\frac{-1}{3}\right]}(Q \mid U)\right)$ is a Hese's canonical plane cubic.

Lemma 2

$$
\begin{aligned}
& \Delta_{1}(Q)=\operatorname{det}\left(\begin{array}{ll}
\vartheta_{[0]}(Q)^{2}, & \vartheta_{\left[\frac{1}{3}\right]}(Q)^{2} \\
\vartheta_{\left[\frac{1}{3}\right]}(Q)^{2}, & \vartheta_{[0]}(Q) \vartheta_{\left[\frac{1}{3}\right]}(Q)
\end{array}\right) \not \equiv 0 \quad \bmod p, \\
& \Delta_{2}(Q)=\operatorname{det}\left(\begin{array}{lll}
\vartheta_{[0]}(Q)^{3}, & \vartheta_{\left[\frac{1}{3}\right]}(Q)^{2}, & \vartheta_{\left[\frac{1}{3}\right]}(Q)^{3} \\
\vartheta_{\left[\frac{1}{3}\right]}(Q)^{3}, & \vartheta_{\left[\frac{1}{3}\right]}(Q)^{3}, & \vartheta_{[0]}(Q)^{3} \\
\vartheta_{\left[\frac{1}{3}\right]}(Q)^{3}, & \vartheta_{[0]}(Q)^{3}, & \vartheta_{\left[\frac{1}{3}\right]}(Q)^{3}
\end{array}\right) \not \equiv 0 \bmod p, \\
& \gamma(Q)=\vartheta_{[0]}(Q) \vartheta_{\left[\frac{1}{3}\right]}(Q)^{2}\left(\vartheta_{[0]}(Q)^{3}+2 \vartheta_{\left[\frac{1}{3}\right]}(Q)^{3}\right)^{-1} \not \equiv 0 \bmod p,
\end{aligned}
$$

for any prime number $p$.

Proof. From the expansions 


$$
\begin{aligned}
& \vartheta_{[0]}(Q)=1+2 Q+\ldots \ldots \\
& \vartheta_{\left[\frac{1}{3}\right]}(Q)=Q^{\frac{1}{9}}\left(1+Q^{\frac{1}{3}}+\ldots \ldots .\right.
\end{aligned}
$$

it follows

$$
\left.\begin{array}{l}
\Delta_{1}(Q)=Q^{\frac{1}{9}}\left(1-Q^{\frac{2}{3}}+\ldots \ldots\right) \\
\Delta_{2}(Q)=1+3 Q^{\frac{2}{3}}+\ldots \ldots \\
\vartheta_{[0]}(Q) \vartheta_{\left[\frac{1}{3}\right]}(Q)^{2}=Q^{\frac{2}{9}}+\ldots \ldots \\
\vartheta_{[0]}(Q)^{3}+2 \vartheta_{\left[\frac{1}{3}\right]}(Q)^{3}=1+\ldots \ldots \\
\gamma(Q) \equiv Q^{\frac{2}{9}}+\ldots \ldots
\end{array}\right\} \not \equiv 0 \quad \bmod p,
$$

Proposition 2. Let $K$ be a field containing $\gamma(Q)$ and $\mathfrak{q}$ be the ideal in $K\left[X_{[0]}, X_{\left[\frac{1}{3}\right]}, X_{\left[\frac{-1}{3}\right]}\right]$ generated by the cubic from

$$
X_{[0]} X_{\left[\frac{1}{3}\right]} X_{\left[\frac{-1}{3}\right]}-\gamma(Q)\left(X_{[0]}^{3}+X_{\left[\frac{1}{3}\right]}^{3}+X_{\left[\frac{-1}{3}\right]}^{3}\right) .
$$

Then $K\left[X_{[0]}, \quad X_{\left[\frac{1}{3}\right]}, \quad X_{\left[\frac{-1}{3}\right]}\right] / \mathfrak{q} \quad$ is canonically isomorphic to $K\left[\vartheta_{[0]}(Q \mid U), \quad \vartheta_{\left[\frac{1}{3}\right]}(Q \mid U)\right.$, $\left.\vartheta_{\left[\frac{-1}{3}\right]}(Q \mid U)\right]$ by the mapp $\left(X_{[0]}, \quad X_{\left[\frac{1}{3}\right]}, \quad X_{\left[\frac{-1}{3}\right]}\right) \rightarrow\left(\vartheta_{[0]}(Q \mid U), \vartheta_{\left[\frac{1}{3}\right]}(Q \mid U), \vartheta_{\left[\frac{-1}{3}\right]}(Q \mid U)\right)$.

Proof. By virtue of Lemma $2 \quad\left(\vartheta_{[0]}(Q \mid U)^{3}, \vartheta_{\left[\frac{1}{3}\right]}(Q \mid U)^{2}, \quad \vartheta_{\left[\frac{-1}{3}\right]}(Q \mid U)^{3}\right)$ is a base of formal theta functions of type $(3 ;[0])$. Since the product $\vartheta_{[0]}(Q \mid U)$ $\vartheta_{\left[\frac{1}{3}\right]}(Q \mid U) \vartheta_{\left[\frac{-1}{3}\right]}(Q \mid U)$ is a formal theta function of type $(3 ;[\mathrm{a}])$, there exists a unique system $\left(\lambda_{[0]}, \lambda_{\left[\frac{1}{8}\right]}, \lambda_{\left[\frac{-1}{3}\right]}\right)$ such that

$$
\begin{aligned}
& \vartheta_{[0]}(Q \mid U) \vartheta_{\left[\frac{1}{3}\right]}(Q \mid U) \vartheta_{\left[\frac{-1}{3}\right]}(Q \mid U) \\
& =\lambda[0] \vartheta_{[0]}(Q \mid U)^{3}+\lambda_{\left[\frac{1}{3}\right]} \vartheta_{\left[\frac{1}{3}\right]}(Q \mid U)^{3}+\lambda_{\left[\frac{-1}{3}\right]} \vartheta_{\left[\frac{-1}{3}\right]}(Q \mid U)^{3} .
\end{aligned}
$$

Replacing $U \rightarrow Q\left(a^{\prime}\right) U\left(a^{\prime}=\frac{1}{3}, \frac{-1}{3}\right)$, we observe that $\lambda_{[0]}=\lambda_{\left[\frac{1}{3}\right]}=\lambda_{\left[\frac{-1}{3}\right]}=\gamma(Q)$.

2.2 We shall generalize Hessian canonical plane cubics to the elliptic curves in projective spaces of even dimension.

Putting

$$
\begin{aligned}
& \left(\begin{array}{ll}
\beta_{[a],[0]}(Q), & \beta_{[a],\left[\frac{1}{3}\right]}(Q) \\
\beta_{\left[a+\frac{1}{3}\right],[0]}(Q), & \beta_{\left[a+\frac{1}{3}\right],\left[\frac{1}{3}\right]}(Q)
\end{array}\right) \\
= & \left(\begin{array}{ll}
\vartheta_{[a]}(Q)_{[-a]}(Q), & \vartheta_{\left[a+\frac{1}{3}\right]}(Q) \vartheta_{\left[-a+\frac{1}{3}\right]}(Q) \\
\vartheta_{\left[a+\frac{1}{3}\right]}(Q) \vartheta_{\left[-a-\frac{1}{3}\right]}(Q), & \vartheta_{\left[a-\frac{1}{3}\right]}(Q) \vartheta_{[-a]}(Q)
\end{array}\right)
\end{aligned}
$$




$$
=\left(\begin{array}{ll}
\vartheta_{[0]}(Q)^{2}, & \vartheta_{\left[\frac{1}{3}\right]}(Q)^{2} \\
\vartheta_{\left[\frac{1}{3}\right]}(Q)^{2}, & \vartheta_{[0]}(Q) \vartheta_{\left[\frac{1}{3}\right]}(Q)
\end{array}\right)^{-1}
$$

we have the next Lemma:

Lemma 3. Let $[a]$ be an element of odd order in $\boldsymbol{Q} / \boldsymbol{Z}$. Then it follows

$$
\operatorname{det}\left(\begin{array}{ll}
\beta_{[a],[0]}(Q), & \beta_{[a],\left[\frac{1}{3}\right]}(Q) \\
\beta_{\left[a+\frac{1}{3}\right],[0]}(Q), & \beta_{\left[a+\frac{1}{3}\right],\left[\frac{1}{3}\right]}(Q)
\end{array}\right) \neq 0 \quad \bmod p
$$

for any prime number $p$.

Proof. Since $\Delta_{1}(Q) \not \equiv 0 \bmod p$, it is sufficient to show that

$$
\begin{aligned}
& \operatorname{det}\left(\begin{array}{ll}
\vartheta_{[a]}(Q)^{2}, & \vartheta_{\left[a+\frac{1}{3}\right]}(Q) \vartheta_{\left[a-\frac{1}{3}\right]}(Q) \\
\vartheta_{\left[a+\frac{1}{3}\right]}(Q)^{2}, & \vartheta_{\left[a-\frac{1}{3}\right]}(Q) \vartheta_{[a]}(Q)
\end{array}\right) \\
& =\vartheta_{\left[a-\frac{1}{3}\right]}(Q)\left(\vartheta_{[a]}(Q)^{3}-\vartheta_{\left[a+\frac{1}{3}\right]}(Q)^{3}\right) \not \equiv 0 \quad \bmod p .
\end{aligned}
$$

From the expansions in $Q$

$$
\begin{aligned}
& \vartheta_{[a]}(Q)= \begin{cases}Q^{a^{2}}+\ldots . . & \text { for } a<\frac{1}{2}, \\
2 Q^{\frac{1}{4}}+\ldots \ldots . & \text { for } a=\frac{1}{2}\end{cases} \\
& \vartheta_{\left[a+\frac{1}{3}\right]}(Q)= \begin{cases}Q^{\left(a+\frac{1}{3}\right)^{3}}+\ldots . . & \text { for } \frac{-1}{2} \leq a<\frac{1}{6}, \\
2 a^{\frac{1}{4}}+\ldots \ldots & \text { for } a=\frac{1}{6}, \\
Q^{\left(1-a-\frac{1}{3}\right)^{2}}+\ldots . . & \text { for } \frac{1}{6}<a \leq \frac{1}{2},\end{cases} \\
& \vartheta_{\left[a-\frac{1}{3}\right]}(Q)= \begin{cases}Q^{\left(a-\frac{1}{3}\right)^{2}}+\ldots \ldots . & \text { for } a=\frac{-1}{6}<a \leq \frac{1}{2}, \\
2 Q^{\frac{1}{4}}+\ldots \ldots & \text { for } a=\frac{-1}{6}, \\
Q^{\left(1+a-\frac{1}{3}\right)^{2}}+\ldots . . & \text { for } \quad \frac{-1}{2} \leq a<\frac{-1}{6},\end{cases}
\end{aligned}
$$

it follows

$$
\vartheta_{\left[a-\frac{1}{3}\right]}(Q) \not \equiv 0 \quad \bmod p \quad\left([a] \neq\left[\frac{-1}{6}\right]\right)
$$




$$
\vartheta_{[a]}(Q)^{3}-\vartheta_{\left[a+\frac{1}{3}\right]}(Q)^{3}= \begin{cases}Q^{3 a^{2}}+\ldots \ldots & \text { for } \frac{-1}{2}<a<0 \\ Q^{3 a^{2}}+\ldots \ldots & \text { for } 0<a<\frac{1}{3} \\ -Q^{3\left(1-a-\frac{1}{3}\right)^{2}} & \text { for } \frac{1}{3}<a<\frac{1}{2} .\end{cases}
$$

This means

$$
\vartheta_{\left[a-\frac{1}{3}\right]}(Q)\left(\vartheta_{[a]}(Q)^{3}-\vartheta_{\left[a+\frac{1}{3}\right]}(Q)^{3} \neq 0 \quad \bmod p\right.
$$

for $[a] \neq\left[\frac{-1}{6}\right],[0],\left[\frac{1}{3}\right]$. On the other hand

$$
\left(\begin{array}{ll}
\beta_{[0],[0]}(Q), & \beta_{[0],\left[\frac{1}{3}\right]}(Q) \\
\beta_{\left[\frac{1}{3}\right],[0]}(Q), & \beta_{\left[\frac{1}{3}\right],\left[\frac{1}{3}\right]}(Q)
\end{array}\right)=\left(\begin{array}{cc}
1 & 0 \\
0 & 1
\end{array}\right),
$$

hance we have Lemma.

For the sake of simplicity we mean by the same symbol $r(Q), \beta_{[a] \cdot[0]}(Q)$, $\beta_{[a],\left[\frac{1}{3}\right]}(Q)$ the images $\gamma(Q) \otimes_{z} 1, \beta_{[a],[0]}(Q) \otimes_{z} 1, \beta_{[a],\left[\frac{1}{3}\right]}(Q) \otimes_{z} 1$ in the tensor product $\boldsymbol{Z}\left[\gamma(Q), \beta_{[a],\left[\frac{1}{3}\right]}(Q)\right]_{\otimes_{Z}} G F(p)$, where $G F(p)$ means the prime field of characteristic $p$.

Theorem 1. Let $n$ be an odd positive integer and $K$ be a field containing $r(Q)$, $\beta_{[a],[0]}(Q), \beta_{[a],\left[\frac{1}{3}\right]}(Q),\left([a] \in \frac{1}{3 n} \boldsymbol{Z} / \boldsymbol{Z}\right) . \quad$ Let $\mathfrak{q}$ be the ideal in $K\left[\left\{X_{[a]}\left|[a] \in \frac{1}{3 n} \boldsymbol{Z}\right| \boldsymbol{Z}\right\}\right]$ generated by the homogeneous elements:

$$
\begin{array}{r}
X_{[0]+[b]} X_{\left[\frac{1}{3}\right]+[b]} X_{\left[\frac{-1}{3}\right]+[b]}-\gamma(Q)\left(X_{[0]+[b]}^{3}+X_{\left[\frac{1}{3}\right]+[b]}^{3}+X_{\left[\frac{-1}{3}\right]+[b]}^{3}\right), \\
X_{[a]+[b]} X_{-[a]+[b]}-\beta_{[a],\left[\frac{1}{3}\right]}(Q) X_{[b]}^{2}-\beta_{[a],[0]}(Q) X_{\left[\frac{1}{3}\right]+[b]} X_{-\left[\frac{1}{3}\right]+[b]} \\
\left([a],[b] \in \frac{1}{3 n} \boldsymbol{Z} / Z\right) .
\end{array}
$$

Then $K\left[\left\{X_{[a]}\left|[a] \in \frac{1}{3 n} \boldsymbol{Z}\right| \boldsymbol{Z}\right\}\right] / q$ is isomorphic to $K\left[\left\{\vartheta_{[a]}(Q \mid U)\left|[a] \in \frac{1}{3 n} \boldsymbol{Z}\right| \boldsymbol{Z}\right\}\right]$ by the map $\rho: X_{[a]} \rightarrow \vartheta_{[a]}(Q \mid U)\left([a] \in \frac{1}{3 n} \boldsymbol{Z} \mid \boldsymbol{Z}\right)$.

Proof. Let $\mathfrak{p}$ be the kernel of $\rho$ in $K\left[\left\{X_{[a]} \mid[a] \in \frac{1}{3 n} \boldsymbol{Z} / \boldsymbol{Z}\right\}\right]$. Then by virtue of Proposition $2 \mathfrak{p}$ is a prime ideal such that $\mathfrak{p}_{0}=\mathfrak{p} \cap K\left[X_{[0]}, X_{\left[\frac{1}{3}\right]}, X_{\left[\frac{-1}{3}\right]}\right]$ 
is a principal ideal $\left(X_{[0]} X_{\left[\frac{1}{3}\right]} X_{\left[\frac{-1}{3}\right]}-\gamma(Q)\left(X_{[0]}^{3}+X_{\left[\frac{1}{3}\right]}^{\mathbf{3}}+X_{\left[\frac{-1}{3}\right]}^{\mathbf{3}}\right)\right)$ and further more $\mathfrak{p}_{0}=\mathfrak{q} \cap K\left[X_{[0]}, X_{\left[\frac{1}{3}\right]}, X_{\left[\frac{-1}{3}\right]}\right]$. Denote by $x_{[a]}$ the image of $X_{[a]}$ in the residue algebra $K\left[\left\{X_{[a]}\left|[a] \in \frac{1}{3 n} \boldsymbol{Z}\right| \boldsymbol{Z}\right\}\right] / \mathfrak{p}_{0}$ and by $\overline{\mathfrak{p}}$ and $\overline{\mathfrak{q}}$ the images of $\mathfrak{p}$ and $\mathfrak{q}$ in $K\left[\left\{x_{[a]} \mid\right.\right.$ $\left.\left.[a] \in \frac{1}{3 n} Z \mid Z\right\}\right]$, respectively. We whall divide the proof into the three steps.

Step $1^{0}$ : Let us first prove $\mathfrak{p} \supset \mathfrak{q}$.

Since $\vartheta_{[a]+[b]}(Q \mid U)=Q(b, b) U(b)^{2} \vartheta_{[a]}(Q \mid Q(b) U)$, it is sufficient to prove that

$$
\begin{aligned}
& \vartheta_{[a]}(Q \mid U) \vartheta_{-[a]}(Q \mid U)=\beta_{[a],[0]}(Q) \vartheta_{[0]}(Q \mid U)^{2} \\
& \quad+\beta_{[a],\left[\frac{1}{3}\right]}(Q) \vartheta_{\left[\frac{1}{3}\right]}(Q \mid U) \vartheta_{\left[\frac{-1}{3}\right]}(Q \mid U) \quad\left([a] \in \frac{1}{3 n} Z \mid Z\right) .
\end{aligned}
$$

The products $\vartheta_{[a]}(Q \mid U) \vartheta_{-[a]}(Q \mid U)\left([a] \in \frac{1}{3 n} \boldsymbol{Z} \mid \boldsymbol{Z}\right)$ are theta functions of type $\left(2\right.$; [0]) and $\left\{\vartheta_{[0]}(Q \mid U)^{2}, \quad \vartheta_{\left[\frac{1}{3}\right]}(Q \mid U) \vartheta_{\left[\frac{-1}{3}\right]}(Q \mid U)\right\}$ is a base of formal theta functions of type $(2 ;[0])$. Therefore, since $\vartheta_{[c]+\left[\frac{1}{3}\right]}(Q \mid U)=Q\left(\frac{1}{3}, \frac{1}{3}\right) U\left(\frac{1}{3}\right)^{2} \vartheta_{[c]}(Q \mid$ $\left.Q\left(\frac{1}{3}\right) U\right), \quad$ we have a system of constants $\left(\lambda_{[a],[0]}, \lambda_{[a],\left[\frac{1}{3}\right]}\right)$ such that

$$
\begin{gathered}
\vartheta_{[a]}(Q \mid U) \vartheta_{-[a]}(Q \mid U)=\lambda_{[a],[0]} \vartheta_{[0]}(Q \mid U)^{2} \\
+\lambda_{[a],\left[\frac{1}{3}\right]} \vartheta_{\left[\frac{1}{3}\right]}(Q \mid U) \vartheta_{\left[\frac{-1}{3}\right]}(Q \mid U) \\
\vartheta_{[a]+\left[\frac{1}{3}\right]}(Q \mid U) \vartheta_{-[a]+\left[\frac{1}{3}\right]}(Q \mid U)=\lambda_{[a],[0]} \vartheta_{\left[\frac{1}{3}\right]}(Q \mid U)^{2} \\
+\lambda_{[a],\left[\frac{1}{3}\right]} \vartheta_{[0]}(Q \mid U) \vartheta_{\left[\frac{-1}{3}\right]}(Q \mid U)
\end{gathered}
$$

Putting $U(c)=1(c \in Q)$, we have $\lambda_{[a],[0]}=\beta_{[a],[0]}(Q)$ and $\lambda_{[a],\left[\frac{1}{3}\right]}=\beta_{[a],\left[\frac{1}{3}\right]}(Q)$. Since $\vartheta_{[a+b]}(Q \mid U)=Q(b, b) U(b) \vartheta_{[a]}(Q \mid Q(b) U)$, we have $\vartheta_{[b]}(Q \mid U) \vartheta_{\left[b+\frac{1}{3}\right]}(Q \mid U) \vartheta_{\left[b-\frac{1}{3}\right]}$ $(Q \mid U)=r(Q)\left(\vartheta_{[b]}(Q \mid U)^{3}+\vartheta_{\left[b+\frac{1}{3}\right]}(Q \mid U)^{3}+\vartheta_{\left[b-\frac{1}{3}\right]}(Q \mid U)^{3}\right.$.

This proves $\mathfrak{p} \supset \mathfrak{q}$.

Step $2^{0}$ : We shall next prove that $\overline{\mathfrak{p}} \otimes_{K} K\left(x_{[0]}, x_{\left[\frac{1}{3}\right]}, x_{\left[-\frac{1}{3}\right]}\right)=\overline{\mathfrak{q}} \otimes_{K} K\left(x_{[0]}, x_{\left[\frac{1}{3}\right]}, x_{\left[\frac{-1}{3}\right]}\right)$, where $K\left(x_{[0]}, x_{\left[\frac{1}{3}\right]}, x_{\left[\frac{-1}{3}\right]}\right)$ is the quotient field of $K\left[x_{[0]}, x_{\left[\frac{1}{3}\right]}, x_{\left[\frac{-1}{3}\right]}\right.$. Let $\xi_{[a]}$ be the image of $x_{[a]}$ in the residue algebra $K\left[\left\{x_{[a]} \mid[a] \in \frac{1}{3 n} \boldsymbol{Z} / \boldsymbol{Z}\right\}\right] / \overline{\mathfrak{q}}$. Then it 
follows

$$
\begin{array}{r}
\xi_{[a]+[b]} \xi_{-[a]+[b]}=\beta_{[a],[0]}(Q) \xi_{[b]}^{2}+\beta_{[a],\left[\frac{1}{3}\right]}(Q) \xi_{[b]+\left[\frac{1}{3}\right]} \xi_{[b]-\left[\frac{1}{3}\right]} \\
\left([a],[b] \in \frac{1}{3 n} \boldsymbol{Z} / \boldsymbol{Z}\right) .
\end{array}
$$

Since

$$
\left(\begin{array}{ll}
\beta_{[0],[0]}(Q), & \beta_{[0],\left[\frac{1}{3}\right]}(Q) \\
\beta_{\left[\frac{1}{3}\right],[0]}(Q), & \beta_{\left[\frac{1}{3}\right],\left[\frac{1}{3}\right]}(Q)
\end{array}\right)=\left(\begin{array}{ll}
1 & 0 \\
0 & 1
\end{array}\right),
$$

the map: $\left(x_{[0]}, x_{\left[\frac{1}{3}\right]}, x_{\left[\frac{-1}{3}\right]}\right) \rightarrow\left(\xi_{[0]}, \xi_{\left[\frac{1}{3}\right]}, \xi_{\left[\frac{-1}{3}\right]}\right)$ induceds an isomorphism of $K\left[x_{[0]}, x_{\left[\frac{1}{3}\right]}, x_{\left[\frac{-1}{3}\right]}\right]$ to $K\left[\xi_{[0]}, \xi_{\left[\frac{1}{3}\right]}, \xi_{\left[\begin{array}{c}-1 \\ 3\end{array}\right]}\right]$. Therefore $K\left[\xi_{[0]}, \xi_{\left[\frac{1}{3}\right]}, \xi_{\left[\frac{-1}{3}\right]}\right]$ is isomorphic to $K\left[\vartheta_{[0]}(Q \mid U), \vartheta_{\left[\frac{1}{3}\right]}(Q \mid U), \vartheta_{\left[\frac{-1}{3}\right]}(Q \mid U)\right] . \quad$ The products of theta functions $\vartheta_{[a]}(Q \mid U) \vartheta_{-[a]}(Q \mid U)$ and $\vartheta_{[a] \pm\left[\frac{1}{3}\right]}(Q \mid U) \vartheta_{[a] \pm\left[\frac{1}{3}\right]}(Q \mid U)\left([a] \in \frac{1}{3 n} \boldsymbol{Z} \mid \boldsymbol{Z}\right)$ are not zero and satisfy

$$
\begin{gathered}
\vartheta_{[a]}(Q \mid U) \vartheta_{-[a]}(Q \mid U)=\beta_{[a],[0]}(Q) \vartheta_{[0]}(Q \mid U)^{2}+\beta_{[a],\left[\frac{1}{3}\right]}(Q) \vartheta_{\left[\frac{1}{3}\right]}(Q \mid U) \vartheta_{-\left[\frac{1}{3}\right]}(Q \mid U) \\
\vartheta_{[a] \pm\left[\frac{1}{3}\right]}(Q \mid U) \vartheta_{-[a] \pm\left[\frac{1}{3}\right]}(Q \mid U)=\beta_{[a],[0]]}(Q) \vartheta_{ \pm\left[\frac{1}{3}\right]}(Q \mid U)^{2} \\
+\beta_{[a],\left[\frac{1}{3}\right]}(Q) \vartheta_{[0]}(Q \mid U) \vartheta_{-\left[\frac{1}{3}\right]}(Q \mid U) \quad\left([a] \in \frac{1}{3 n} \boldsymbol{Z} \mid \boldsymbol{Z}\right) .
\end{gathered}
$$

Hence the elements

$$
\beta_{[a],[0]}(Q) \xi_{[0]}^{2}+\beta_{[a],\left[\frac{1}{3}\right]}(Q) \xi_{\left[\frac{1}{3}\right]^{5}-\left[\frac{1}{3}\right]}, \beta_{[a],[0]}(Q) \xi_{\left[\frac{1}{3}\right]}^{2}+\beta_{] a],\left[\frac{1}{3}\right]}(Q) \xi_{[0]} \xi_{-\left[\frac{1}{3}\right]}
$$

are not zero in $K\left[\xi_{[0]}, \xi_{\left[\frac{1}{3}\right]}, \xi_{-\left[\frac{1}{3}\right]}\right]$. Therefore $\xi_{[a]} \xi_{-[a]}$ and $\xi_{[a] \pm\left[\frac{1}{3}\right]} \xi_{-[a] \pm\left[\frac{1}{3}\right]}$ are non-zero elements in $K\left[\xi_{[0]}, \quad \xi_{\left[\frac{1}{3}\right]}, \xi_{-\left[\frac{1}{3}\right]}\right]$ and thus $\xi_{[a]}\left([a] \in \frac{1}{3 n} \boldsymbol{Z} / \boldsymbol{Z}\right)$ are nonzero divisor in the algebra $K\left[\left\{\xi_{[a]}\left|[a] \in \frac{1}{3 n} \boldsymbol{Z}\right| \boldsymbol{Z}\right\}\right]$. We shall prove that there exists a system of non-zero elements $\left\{f_{[a],[b]}\right\}$ in the field $K\left(\xi_{[0]}, \xi_{\left[\frac{1}{3}\right]}, \xi_{\left[\frac{-1}{3}\right]}\right]$ such that $\xi_{l[a]}=f_{l[a],[a]^{[a]}} \xi_{[a]}^{l}\left(\left[a \frac{1}{3 n} \boldsymbol{Z} / \boldsymbol{Z} ; l=1,2,3, \ldots \ldots\right)\right.$. Assume $\xi_{h[a]}=f_{h[a],[a]^{[a]}} \xi^{h}$ 
$(1 \leq h \leq l)$ with non-zero elements $f_{h[a],[a]}$ in $K\left(\xi_{[0]} \xi_{\left[\frac{1}{3}\right]} \xi_{\left[\frac{-1}{3}\right]}\right]$.

Then it follows:

$$
\begin{aligned}
\xi_{(h+1)[a]} \xi_{(h-1)[a]} & =\xi_{h[a]+[a]} \xi_{h[a]-[a]} \\
& =\beta_{[a],[0]}(Q) \xi_{h[a]}^{2}+\beta_{[a],\left[\frac{1}{3}\right]}(Q) \xi_{h[a] \pm\left[\frac{1}{3}\right]} \xi_{-h[a]-\left[\frac{1}{3}\right]}
\end{aligned}
$$

and thus

$$
\begin{gathered}
\xi_{(h+1)[a]} f_{(h-1)[a],[a]^{[a]}} \xi^{h-1}=\left(\beta_{[a],[0]}(Q)+\beta_{[a],\left[\frac{1}{3}\right]}(Q) \frac{\xi_{h[a]+\left[\frac{1}{3}\right]^{\prime} h[a]-\left[\frac{1}{3}\right]^{2}-h[a]} \xi^{2}}{\xi_{h[a]}^{2}-h[a]} f_{h[a],[a][a]}^{2} \xi^{2 h}\right. \\
\xi_{(h+1)[a]}=f_{(h-1)[a]}^{-1}\left(\beta_{[a],[0]}(Q)+\beta_{[a],\left[\frac{1}{3}\right]}(Q) \frac{\xi_{h[a]+\left[\frac{1}{3}\right]_{h[a]-\left[\frac{1}{3}\right]^{2}-h[a]} \xi^{2}}}{\xi_{h[a]}^{2} \xi^{2}-h[a]} \xi_{[a]}^{h+1}\right.
\end{gathered}
$$

This proves $\xi_{l[a]}=f_{l[a],[a]} \xi_{[a]}\left([a] \in \frac{1}{3 n} \boldsymbol{Z} \mid \boldsymbol{Z} ; l=1,2, \ldots \ldots\right)$. Let $[a]$ be a generator of $\frac{1}{3 n} \boldsymbol{Z} / \boldsymbol{Z}$. Then, since the theta function $\vartheta_{[a]}(Q \mid U)^{l}$ is a formal theta function of type $(l ; l[a])$, the theta functions $\vartheta_{[a]}(Q \mid U), \vartheta_{[a]}(Q \mid U)^{2}, \ldots \ldots$. $\vartheta_{[a]}(Q \mid U)^{n} \quad$ are linearly independent over $K\left(\vartheta_{[0]}(Q \mid U), \vartheta_{\left[\frac{1}{3}\right]}(Q \mid U), \vartheta_{\left[-\frac{1}{3}\right]}(Q \mid U)\right)$. Hence $\xi_{[a]} \xi_{[a]}^{2}, \ldots . ., \xi_{[a]}^{n}$ are linearly independent over $K\left(\xi_{[0]}, \xi_{\left[\frac{1}{3}\right]}, \xi_{-\left[\frac{1}{3}\right]}\right)$ and the equation $X^{n}-f_{n[a],[a]}^{-1} \xi_{n[a]}=0$ is an irreducible equation over $K\left(\xi_{[0]}\right.$, $\left.\xi_{\left[\frac{1}{3}\right]}, \xi_{-\left[\frac{1}{3}\right]}\right)$. This means that the algebra $K\left(\xi_{[0]}, \xi_{\left[\frac{1}{3}\right]}, \quad \xi_{\left[-\frac{1}{3}\right]}\right)\left[\left\{\xi_{[a]} \mid[a] \in \frac{1}{3 n} \boldsymbol{Z} / \boldsymbol{Z}\right\}\right]$ is isomorphic to $K\left(\xi_{[0]}, \quad \xi_{\left[\frac{1}{8}\right]}, \quad \xi_{\left[\frac{-1}{3}\right]}\right)[X] /\left(X^{n}-f_{n[a],[a]^{\xi} n[a]}^{-1}\right)$ and thus isomorphic to $K\left(\vartheta_{[0]}(Q \mid U), \quad \vartheta_{\left[\frac{1}{3}\right]}(Q \mid U), \quad \vartheta_{\left[\frac{-1}{3}\right]}(Q \mid U)\right)\left[\left\{\vartheta_{[a]}(Q \mid U)\left|[a] \in \frac{1}{3 n} \boldsymbol{Z}\right| \boldsymbol{Z}\right\}\right]$. This proves

$$
\overline{\mathfrak{p}} \otimes_{K} K\left(x_{[0]}, x_{\left[\frac{1}{3}\right]}, x_{\left[\frac{-1}{3}\right]}\right)=\overline{\mathfrak{q}} \otimes_{K} K\left(x_{[0]}, x_{\left[\frac{1}{3}\right]}, x_{\left[-\frac{1}{3}\right]}\right) .
$$

Step $3^{\circ}$ : Let $F$ be any non-zero element in $\bar{p}$. Then from Step $2^{\circ}$ there exists a non-zero element $H$ in $K\left[x_{[0]}, x_{\left[\frac{1}{3}\right]}, x_{\left[\frac{-1}{3}\right]}\right]$ such that $H F \in \overrightarrow{\mathfrak{q}}$. Therefore, if $\bar{q}$ is a primary ideal belonging to the prime ideal $\bar{p}$ then the element $F$ itself belongs to $\overline{\mathfrak{q}}$ because $\bar{p} \cap K\left[x_{[0]}, x_{\left[\frac{1}{3}\right]^{3}}, x_{\left[\frac{-1}{3}\right]}\right]=(0)$. Hence it is sufficient to show that the ideal $\mathfrak{q}$ is a primary ideal belonging to the prime ideal $\mathfrak{p}$. Let $S_{3 n}$ be 
the affine $(3 n+1)$-affine space with the system $\left(\ldots \ldots, X_{[a]}, \ldots ..\right)$ of coordinates and $V(\mathfrak{p})$ (resp. $V(\mathfrak{q})$ ) be the affine variety corresponding to the ideal $\mathfrak{p}$ (resp. $\mathfrak{q}$ ). Since $\mathfrak{p}$ is a prime ideal, it is sufficient to show $V(\mathfrak{p}) \supset V(\mathfrak{q})$. Let $\sigma_{-1}$ and $\sigma_{[b]}$ be the linear transformation of $S_{3 n+1}$ such that $\sigma_{-1}\left(X_{[c]}\right)=X_{-[c]}$ and $\sigma_{[b]}\left(X_{[c]}\right)$ $=X_{[b]+[c]}\left([b],[c] \in \frac{1}{3 n} \boldsymbol{Z} / \boldsymbol{Z}\right)$. Then $\sigma_{-1}$ and $\sigma_{[b]}$ leave the affine varieties $V(\mathfrak{p})$ and $V(\mathfrak{q})$ invariant and the origin $(0, \ldots \ldots, 0)$ belongs to $V(\mathfrak{p})$ and $V(\mathfrak{q})$. Let $\left(\ldots \ldots, \eta_{[c]}, \ldots ..\right)$ be any point on $V(\mathfrak{q})$ which is different with the origin $(0$, $0)$. Let us prove that belongs to $V(\mathfrak{p})$. After the transformation by an element $\sigma_{[c]}$ we may assume without loss of generality that $\eta_{[0]} \neq 0$ and $\eta_{\left[\frac{1}{3}\right]} \neq 0$, because from $\eta_{[0]} \eta_{\left[\frac{1}{3}\right]} \eta_{\left[\frac{-1}{3}\right]}=\gamma(Q)\left(\eta_{[0]}^{3}+\eta_{\left[\frac{1}{3}\right]}^{3}+\eta_{\left[\frac{-1}{3}\right]}^{3}\right)$ the inequality $\eta_{[0]} \neq 0$ implies $\eta_{\left[\frac{1}{3}\right]} \neq 0$ or $\eta_{\left[\frac{-1}{3}\right]} \neq 0$.

Since

$$
\left(\begin{array}{ll}
\eta_{[a]} \eta_{-[a]}, & \eta_{\left[a+\frac{1}{3}\right]^{\eta}-\left[a+\frac{1}{3}\right]} \\
\eta_{\left[a+\frac{1}{3}\right]^{\eta}-\left[a+\frac{1}{3}\right]}, & \eta_{\left[a-\frac{1}{3}\right]^{\eta}-[a]}
\end{array}\right)=\left(\begin{array}{cc}
\beta_{[a],[0]}(Q) & \beta_{[a],\left[\frac{1}{3}\right]}(Q) \\
\beta_{\left[a+\frac{1}{3}\right],[0]}(Q) \beta_{\left[a+\frac{1}{3}\right],\left[\frac{1}{3}\right]}(Q)
\end{array}\right)\left(\begin{array}{cc}
\eta_{[0]}^{2}, & \eta_{\left.\left[\frac{1}{3}\right]\right]^{2}\left[\frac{-1}{3}\right]} \\
\eta_{\left[\frac{1}{3}\right]}^{2}, & \eta_{[0]} \eta_{\left[\frac{-1}{3}\right]}
\end{array}\right)
$$

and

$$
\operatorname{det}\left(\begin{array}{ll}
\beta_{[a],[0]}(Q), & \beta_{[a],\left[\frac{1}{3}\right]}(Q) \\
\beta_{\left[a+\frac{1}{3}\right],[0]}(Q), & \beta_{\left[a+\frac{1}{3}\right],\left[\frac{1}{3}\right]}(Q)
\end{array}\right) \neq 0
$$

it follows that $\eta_{[a]} \eta_{-[a]} \neq 0$ or $\eta_{\left[a+\frac{1}{3}\right]} \eta_{-\left[a+\frac{1}{3}\right]} \neq 0$. For each element $[a]$ we choose an element $\hat{[a}]$ such that $[\hat{a}] \equiv[a] \bmod \frac{1}{3} \boldsymbol{Z} / \boldsymbol{Z}$ and $\xi_{[\hat{a}]^{\xi}-[\hat{a}]} \neq 0$. We shall first show that there exist non-zero elements $g_{[\widehat{l a}], \widehat{[a]}}$ in the field $K\left(\eta_{[0]}, \eta_{\left[\frac{1}{3}\right]}, \eta_{\left[\frac{-1}{3}\right]}\right)$ such that $\eta_{[\hat{l a}]}=g_{[\widehat{l a}], \hat{[a]} \eta_{\hat{[a]}}^{l}}\left([a] \in \frac{1}{3 n} \boldsymbol{Z} / \boldsymbol{Z} ; l=1,2,3, \ldots \ldots\right)$. Assume $\eta_{[\widehat{h a}]}=$ $g_{[\widehat{h a}], \hat{a}]_{[a]}} \eta_{[a]}^{n}\left(\left[a \frac{1}{3 n} \boldsymbol{Z} \mid \boldsymbol{Z} ; h=1,2,3, \ldots \ldots, l\right) . \quad\right.$ Then it follows that for each element $[a]$ in $\frac{1}{3 n} \boldsymbol{Z} / \boldsymbol{Z}$ there exist $\left[a^{\prime}\right]$ and $\left[b^{\prime}\right]$ in $\frac{1}{3 n} \boldsymbol{Z} / \boldsymbol{Z}$ such that 


$$
\begin{aligned}
& \left.\eta_{[(\widehat{l+1}) a]^{\eta}[(\widehat{l-1) a]}}=\eta_{\left[a+a^{\prime}\right]+\left[l a+b^{\prime}\right]}\right]_{-\left[a+a^{\prime}\right]+\left[l a+b^{\prime}\right]} \\
& =\beta_{\left[a+a^{\prime}\right],[0]}(Q) \eta_{\left[l a+b^{\prime}\right]}^{2}+\beta_{\left[a+a^{\prime}\right],\left[\frac{1}{3}\right]}(Q) \eta_{\left[l a+b^{\prime}+\frac{1}{3}\right]} \eta_{\left[l a+b^{\prime}-\frac{1}{3}\right]} \\
& =\left[\beta_{\left[a+a^{\prime}\right],[0]}(Q)\left(\frac{\left.\eta_{\left[l a+b^{\prime}\right]}\right]^{\eta}-[\hat{l a}]}{\eta_{[\hat{l a}]^{\eta}-[\hat{l a}]}}\right)^{2}\right. \\
& \left.+\beta_{\left[a+a^{\prime}\right],\left[\frac{1}{3}\right]}(Q) \frac{\eta_{\left[l a+b /+\frac{1}{3}\right]^{\eta}\left[l a+b /-\frac{1}{3}\right]^{\eta^{2}}-[l a]}}{\left.\eta_{[l a]}^{2}\right]_{-[l a]}^{2}}\right] \eta_{[\hat{l} a]}^{2} .
\end{aligned}
$$

From the assumption of the inductive we have

$$
\eta_{[(l+1) a]}=g_{[(\hat{l+1}) a], \widehat{[a]}} \eta_{[a]}^{l+1}
$$

with a non-zero element $g_{[(\hat{l+1}) a], \hat{[a]}}$ If $\eta_{[a]^{\eta}-[a]} \neq 0$, it follows

$$
\begin{aligned}
\eta_{l[a]} & =\frac{\eta_{[l a]^{\eta}-[\hat{l a}]}}{\eta_{[\hat{l a}]^{\eta}-[\hat{l a}]} \eta_{[\hat{l a}]}} \\
& =\frac{\eta_{[l a]^{\prime}-[\hat{l a}]}}{\eta_{[l a]^{\eta}-[\hat{l a}]}} g_{[\hat{l a}],[\hat{a}]}\left(\frac{\eta_{[\hat{a}]^{\eta}-[\hat{a}]}}{\eta_{[a]} \eta_{-[a]}}\right)^{l} \eta_{[a]}^{l}, \\
\eta_{l[a]} & =g_{l[a] .[a]} \eta_{[a]}^{l} .
\end{aligned}
$$

The coefficients $g_{l[a],[a]}$ are uniquely determined provided $\eta_{[a]} \eta_{-[a]} \neq 0$. Let us assume that $[a]$ is a generator of $\frac{1}{3 n} \boldsymbol{Z} / \boldsymbol{Z}$ such that $\eta_{[a]} \eta_{-[a]} \neq 0$. Then the above result means that the map: $f_{l[a],[a]} \rightarrow g_{l[a],[a]}(l=1,2,3, \ldots \ldots)$ is a specialization on the specialization $\left(\xi_{[0]}, \xi_{\left[\frac{1}{3}\right]}, \xi_{\left[\frac{-1}{3}\right]}\right) \rightarrow\left(\eta_{[0]}, \eta_{\left[\frac{1}{3}\right]}, \eta_{\left[\frac{-1}{3}\right]}\right)$ over $K$. Therefore the map $\xi=\left(\ldots \ldots, \xi_{[b]}, \ldots ..\right) \rightarrow \eta=\left(\ldots \ldots, \eta_{[b]}, \ldots ..\right)$ is a specialization over $K$ and thus $\eta$ belongs to $V(\mathfrak{p})$. This completes the long proof of Theorem.

$\S 3$. Proof of the main theorem

3.1 In order to prove the main theorem we shall be first concerned with product of elliptic case. 
Putting

$$
\begin{aligned}
& \vartheta_{\left(\left[a_{1}\right], \ldots \ldots,\left[a_{r}\right]\right)}\left(Q_{1}, \ldots \ldots, Q_{r} \mid U_{1}, \ldots \ldots, U_{r}\right)=\prod_{i=1}^{r} \vartheta_{\left[a_{i}\right]}\left(Q_{i} \mid U_{i}\right), \\
& \vartheta_{\left(\left[a_{1}\right], \ldots \ldots,\left[a_{r}\right]\right)}\left(Q_{1}, \ldots \ldots, Q_{r}\right) \quad=\prod_{i=1}^{r} \vartheta_{\left[a_{i}\right]}\left(Q_{i}\right) \\
& \gamma_{\left(\left[a_{1}{ }^{\prime}\right], \ldots \ldots,\left[a_{r}{ }^{\prime}\right]\right),\left(\left[c_{1}{ }^{\prime}\right], \ldots \ldots .,\left[c_{r}{ }^{\prime}\right]\right)}\left(Q_{1}, \ldots \ldots, Q_{r}\right)=\prod_{j=1}^{r} \gamma_{\left[a_{i}{ }^{\prime}\right],\left[c_{i}{ }^{\prime}\right]}\left(Q_{i}\right) \\
& \beta_{\left(\left[a_{1}\right], \ldots \ldots,\left[a_{r}\right]\right),\left(\left[c_{1}+\right], \ldots \ldots,\left[c_{r}+\right]\right)}\left(Q_{1}, \ldots \ldots, Q_{r}\right)=\prod_{i=1}^{r} \beta_{\left[a_{i}\right],\left[c_{i}^{+}\right]}\left(Q_{i}\right) \\
& \left(\left[a_{i}\right] \in Q / Z, \quad\left[a_{i}^{\prime}\right]\left[c_{i}{ }^{\prime}\right] \in \frac{1}{3} Z / Z, \quad\left[c^{+}\right] \in\left\{[0],\left[\frac{1}{3}\right]\right\}\right),
\end{aligned}
$$

we shall show the next result.

Theorem 2. Let $G$ be a subgroup in $\boldsymbol{Q}^{r} / \boldsymbol{Z}^{r}$ such that $G \supset H$ and $[G: H]$ is odd. Let $K$ be a field containing $\gamma_{\left[a^{\prime}\right],\left[c^{\prime}\right]}\left(Q_{1}, \ldots \ldots, Q_{r}\right), \beta_{[a],\left[c^{+}\right]}\left(Q_{1}, \ldots \ldots, Q_{r}\right)\left(\left[\boldsymbol{a}^{\prime}\right] \in G\right.$; $\left.\left[\boldsymbol{a}^{\prime}\right],\left[\boldsymbol{c}^{\prime}\right] \in H ;\left[\boldsymbol{c}^{+}\right] \in H^{+}\right)$and $\mathfrak{q}$ be the ideal in $K\left[\left\{X_{[\boldsymbol{a}]} \mid[\boldsymbol{a}] \in G\right\}\right]$ generated by the homogeneous elements

$$
\begin{array}{r}
X_{\left[a^{\prime}\right]+[b]} X_{-\left[\boldsymbol{a}^{\prime}\right]+[b]} X_{1 b]}-\sum_{\left[\boldsymbol{c}^{\prime}\right] \in H^{\prime}} \gamma_{\left[\boldsymbol{a}^{\prime}\right],\left[\boldsymbol{c}^{\prime}\right]}\left(Q_{1}, \ldots \ldots, Q_{r}\right) X_{\left[\boldsymbol{c}^{\prime}\right]+[b]}^{3} \\
X_{[a]+[b]} X_{-[a]+[b]}-\sum_{\left[\boldsymbol{c}^{+}\right] \in H^{+}} \beta_{[a],\left[c^{+}\right]}\left(Q_{1}, \ldots \ldots, Q_{r}\right) X_{[b]+\left[\boldsymbol{c}^{+}\right]} X_{[b]-\left[\boldsymbol{c}^{+}\right]} \\
\left([\boldsymbol{a}],[\boldsymbol{b}] \in G ;\left[\boldsymbol{a}^{\prime}\right] \in H\right)
\end{array}
$$

Then the map $\rho: X_{[a]} \rightarrow \vartheta_{[a]}\left(Q_{1}, \ldots \ldots, Q_{r} \mid U_{1}, \ldots \ldots, U_{r}\right)([\boldsymbol{a}] \in G)$ induces an isomorphism of $K\left[\left\{X_{[a]} \mid[\boldsymbol{a}] \in G\right\}\right] / \mathfrak{q} \quad$ onto $\left.K\left[\vartheta_{[\boldsymbol{a}]}\left(Q_{1}, \ldots \ldots, Q_{r} \mid U_{1} \ldots \ldots, U_{r}\right) \mid[\boldsymbol{a}] \in G\right\}\right]$.

Proof. Step $1^{\circ}$. Let us first prove Theorem under the assumption that Theorem is valid for the special case $\frac{1}{3 n} \boldsymbol{Z}^{r} \mid \boldsymbol{Z}^{r}$ with an odd $n$. Let $n$ be an odd positive integer such that $G \subset \frac{1}{3 n} \boldsymbol{Z}^{r} \mid \boldsymbol{Z}^{r}, K^{\prime}$ be the field $K\left(\left\{\beta_{[a],\left[c^{+}\right]}\left(Q_{1}\right.\right.\right.$, $\left.\left.\left.Q_{r}\right) \mid[\boldsymbol{a}] \in \frac{1}{3 n} \boldsymbol{Z}^{r} / \boldsymbol{Z}^{r}, \quad[\boldsymbol{c}] \in H^{+}\right\}\right)$and $\tilde{\mathfrak{q}}$ be the ideal in $K^{\prime}\left[\left\{X_{[\boldsymbol{a}]} \mid[\boldsymbol{a}] \in\right.\right.$ $\left.\left.\frac{1}{3 n} \boldsymbol{Z}^{r} \mid \boldsymbol{Z}^{r}\right\}\right]$ generated by the homogeneous elements:

$$
X_{\left[a^{\prime}\right]+[b]} X_{-\left[a^{\prime}\right]+[b]} X_{[b]}-\sum_{\left[c^{\prime}\right] \in H} \gamma_{\left[a^{\prime}\right],\left[c^{\prime}\right]}\left(Q_{1}, \ldots \ldots, Q_{r}\right) X_{\left[c^{\prime}\right]+[b]}^{3},
$$




$$
\begin{array}{r}
X_{[a]+[b]} X_{-[a]+[b]}-\sum_{\left[c^{+}\right] \in H^{+}} \beta_{[a],\left[c^{+}\right]}\left(Q_{1}, \ldots \ldots, Q_{r}\right) X_{\left[\boldsymbol{c}^{+}\right]+[b]} X_{-\left[\boldsymbol{c}^{+}\right]+[\boldsymbol{b}]} \\
\left([\boldsymbol{a}],[\boldsymbol{b}] \in \frac{1}{3 n} \boldsymbol{Z}^{r} / \boldsymbol{Z}^{r} ;\left[\boldsymbol{a}^{\prime}\right] \in H\right)
\end{array}
$$

Then it follows that $\mathfrak{q} \otimes_{k} K^{\prime}=\tilde{\mathfrak{q}} \cap K^{\prime}\left[\left\{X_{[\boldsymbol{a}]} \mid[\boldsymbol{a}] \in G\right\}\right]$. From the assumption the $\operatorname{map} \rho: X_{[a]} \rightarrow \vartheta_{[a]}\left(Q_{1}, \ldots \ldots, Q_{r} \mid U_{1}, \ldots \ldots, U_{r}\right)\left([\boldsymbol{a}] \in \frac{1}{3 n} \boldsymbol{Z}^{r} / \boldsymbol{Z}^{r}\right)$ induces an a isomorpihism of $K^{\prime}\left[\left\{X_{[a]}\left|[\boldsymbol{a}] \in \frac{1}{3 n} \boldsymbol{Z}^{r}\right| \boldsymbol{Z}^{r}\right\}\right] / \tilde{q}$ and $K^{\prime}\left[\left\{\vartheta_{[\boldsymbol{a}]}\left(Q_{1}, \ldots \ldots, Q_{r} \mid U_{1}\right.\right.\right.$, $\left.\left.\left.\ldots \ldots, U_{r}\right)\left|[\boldsymbol{\alpha}] \in \frac{1}{3 n} \boldsymbol{Z}^{r}\right| \boldsymbol{Z}^{r}\right\}\right]$, hence the restriction of $\rho$ on $K^{\prime}\left[\left\{X_{[a]} \mid[\boldsymbol{a}] \in G\right\}\right]$ induces an isomorphism of $K^{\prime}\left[\left\{X_{[a]} \mid[\boldsymbol{a}] \in G\right\}\right] / \mathfrak{q}$ onto $K\left[\left\{\vartheta_{[a]}\left(Q_{1}, \ldots \ldots, Q_{r} \mid U_{1}, \ldots \ldots\right.\right.\right.$, $\left.\left.\left.U_{r}\right) \mid[\boldsymbol{\alpha}] \in G\right\}\right]$.

Step $2^{\circ}$. We shall prove Theorem for the case $G=\frac{1}{3 n} \boldsymbol{Z}^{r} / \boldsymbol{Z}^{r}$. Let $\mathfrak{p}_{i}$ be the kernel of the morphism $\rho_{i}: K\left[\left\{X_{i,[a]}\left|[a] \in \frac{1}{3 n} \boldsymbol{Z}\right| \boldsymbol{Z}\right\}\right]$ onto $K\left[\left\{\vartheta_{[a]}\left(Q_{i} \mid U_{i}\right) \mid\right.\right.$ $\left.\left.[a] \in \frac{1}{3 n} \boldsymbol{Z} \mid \boldsymbol{Z}\right\}\right]$ such that $\rho_{i}\left(X_{i,[a]}\right)=\vartheta_{[a]}\left(Q_{i} \mid U_{i}\right)$ and $\mathfrak{p}$ be the kernel of the morphism $\rho: K\left[\left\{X_{[a]}\left|[\boldsymbol{a}] \in \frac{1}{3 n} \boldsymbol{Z}^{r}\right| \boldsymbol{Z}^{r}\right\}\right]$ onto $K\left[\left\{\vartheta_{[a]}\left(Q_{1}, \ldots \ldots, Q_{r} \mid U_{1}, \ldots \ldots, U_{r}\right) \mid\right.\right.$ $\left.\left.[\boldsymbol{a}] \in \frac{1}{3 n} \boldsymbol{Z}^{r} \mid \boldsymbol{Z}^{r}\right\}\right]$ such that $\rho\left(X_{[a]}\right)=\vartheta_{[a]}\left(Q_{1}, \ldots \ldots, Q_{r} \mid U_{1}, \ldots \ldots, U_{r}\right)([\boldsymbol{a}] \in$ $\left.\frac{1}{3 n} \boldsymbol{Z}^{r} \mid \boldsymbol{Z}^{r}\right)$. Let $\lambda$ be the morphism of $K\left[\left\{X_{[a]}\left|[\boldsymbol{a}] \in \frac{1}{3 n} \boldsymbol{Z}^{r}\right| \boldsymbol{Z}^{r}\right\}\right]$ into $K\left[\left\{X_{i,[a]}\left|1 \leq i \leq r ;[a] \in \frac{1}{3 n} \boldsymbol{Z}\right| \boldsymbol{Z}\right\}\right]$ such that $\lambda\left(X_{\left(\left[a_{1}\right], \ldots \ldots,\left[a_{r}\right]\right)}\right)=X_{1,\left[a_{1}\right]} \ldots \ldots . X_{r,\left[a_{r}\right]}$ and $\mathfrak{p}^{*}$ be the inverse image of the ideal $\sum_{i=1}^{r} K\left[\left\{X_{l,[a]}\left|l \neq i ;[a] \in \frac{1}{3 n} \boldsymbol{Z}\right| \boldsymbol{Z}\right\}\right] \mathfrak{p}_{i}$ by the morphism $\lambda$. Then from the definitions it follows that $\mathfrak{p}^{*}$ coincides with $\mathfrak{p}$. From the definitions of $\gamma_{\left[a^{\prime}\right],\left[b^{\prime}\right]}\left(Q_{1}, \ldots \ldots, Q_{r}\right)$ and $\beta_{[a],\left[c^{+}\right]}\left(Q_{1}, \ldots \ldots, Q_{r}\right)$ the ideal $\mathfrak{q}$ is contained in $\mathfrak{p}$, hence it is sufficient to show that $\mathfrak{q} \supset \mathfrak{p}=\mathfrak{p}^{*}$. Since

$$
\begin{gathered}
X_{\left(\left[a_{1}\right], \ldots \ldots,\left[a_{r}\right]\right)} X_{\left(\left[b_{1}\right] \ldots \ldots,\left[b_{r}\right]\right)} \\
\equiv \sum_{\left[c^{+}\right] \in H^{+}} \prod_{i=1}^{r} \beta\left[\frac{a_{i}-b_{i}}{2}\right],\left[c_{i}^{+}\right] \\
X_{\left(-\left[c_{1}+\right], \ldots \ldots,-\left[c_{r}\right]\right)+\left(\left[\frac{a_{1}+b_{1}}{2}, \ldots \ldots .,\left[\frac{a_{r}+b^{+}}{2}\right]\right)\right.}\left(Q_{i}\right) X_{\left(\left[c_{1}^{+}\right], \ldots \ldots .\left[c_{r}^{+}\right]\right)+\left(\left[\frac{a_{1}+b_{1}}{2}\right], \ldots \ldots .,\left[\frac{a_{r}+b_{r}}{-2}\right]\right)} \bmod q
\end{gathered}
$$


and $\beta_{[a],\left[c^{+}\right]}\left(Q_{i}\right)=\beta_{-[a],\left[c^{+}\right]}\left(Q_{i}\right)$, we may conclude that if $\left\{\left[a_{i}\right],\left[b_{i}\right]\right\}=\left\{\left[c_{i}\right],\left[d_{i}\right]\right\}$ $(1 \leq i \leq r)$, it follows

$$
X_{\left(\left[a_{1}\right], \ldots \ldots,\left[a_{r}\right]\right)} X_{\left(\left[b_{1}\right], \ldots \ldots .\left[b_{r}\right]\right)} \equiv X_{\left(\left[c_{1}\right], \ldots \ldots,\left[c_{r}\right]\right)} X_{\left(\left[d_{1}\right], \ldots \ldots,\left[d_{r}\right]\right)} \quad \bmod \mathfrak{q}
$$

This means that $\mathfrak{q}$ contains the kernel of $\lambda$. By virtue of Theorem 1 the ideal $\mathfrak{p}_{i}$ is generated by

$$
\begin{array}{r}
X_{i,\left[a^{\prime}\right]+[b]} X_{i,-\left[a^{\prime}\right]+[b]} X_{i,[b]}-\sum_{\left[c^{\prime}\right] \in \frac{1}{3} Z / Z} \gamma_{\left[a^{\prime}\right],\left[c^{\prime}\right]}\left(Q_{i}\right) X_{i,\left[c^{\prime}\right]}^{\mathbf{s}} \\
X_{i,[a]+[b]} X_{i,-[a]+[b]}-\sum_{\left[c^{+}\right]=\left\{[0],\left[\frac{1}{3}\right]\right\}} \beta_{[a],\left[c^{+}\right]}\left(Q_{i}\right) X_{i,-\left[c^{+}\right]+[b]} X_{i,-\left[c^{+}\right]+[b]} \\
\left(\left[a^{\prime}\right] \in \frac{1}{3} \boldsymbol{Z} / \boldsymbol{Z} ;[a],[b] \in \frac{1}{3 n} \boldsymbol{Z} / \boldsymbol{Z}\right) .
\end{array}
$$

Since

$$
\begin{aligned}
& \gamma_{\left([0], \ldots \ldots,[0],\left[a_{i}{ }^{\prime}\right],[0], \ldots \ldots,[0]\right),\left(\left[b_{1}{ }^{\prime}\right], \ldots \ldots,\left[b_{r}\right]\right)}\left(Q_{1}, \ldots \ldots, Q_{r}\right)=\gamma_{\left[a_{i}^{\prime}\right],\left[b_{i}{ }^{\prime}\right]}\left(Q_{i}\right) \\
& \beta_{\left([0], \ldots \ldots,[0],\left[a_{i}\right],[0], \ldots \ldots .,[0]\right),\left(\left[c_{r}+\right], \ldots \ldots,\left[c_{r}+\right]\right)}\left(Q_{1}, \ldots \ldots, Q_{r}\right)=\beta_{\left[a_{i}\right],\left[c_{i}^{+}\right]}\left(Q_{\imath}\right) \\
& \left(\left[a_{i}{ }^{\prime}\right],\left[b_{i}{ }^{\prime}\right] \in \frac{1}{3} \boldsymbol{Z}\left|\boldsymbol{Z} ;\left[a_{i}\right] \in \frac{1}{3 n} \boldsymbol{Z}\right| \boldsymbol{Z},\left[c_{i}{ }^{+}\right] \in\left\{[0],\left[\frac{1}{3}\right]\right\}\right),
\end{aligned}
$$

the image of $\mathfrak{q}$ by $\lambda$ generates the ideal $\sum_{i=1}^{r} K\left[\left\{X_{l_{,[}[a]}\left|l \neq i,[a] \in \frac{1}{3 n} \boldsymbol{Z}\right| \boldsymbol{Z}\right\}\right] \mathfrak{p}_{i}$ and thus $\subset \mathfrak{p}^{*} \mathfrak{q}$. This completes the proof of theorem.

3.2 By virtue of Lemma 2 for any prime number $p$ it follows

$$
\begin{aligned}
& \operatorname{det}\left(\vartheta_{\left(\left[a_{1}^{\prime}+b_{1}^{\prime}\right], \ldots \ldots,\left[a_{\left.\left.r^{\prime}+b_{r}\right]\right)}^{3}\right.\right.}\left(Q_{1}, \ldots \ldots, Q_{r}\right)\right)_{H \times H}
\end{aligned}
$$

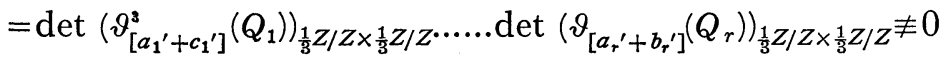

$\operatorname{det}\left(\vartheta_{\left(\left[a_{1}+\right]+\left[b_{1}+\right], \ldots \ldots,\left[a_{r}+\right]+\left[b_{r}+\right]\right)}\left(Q_{1}, \ldots \ldots, Q_{r}\right) \vartheta_{\left(-\left[a_{1}+\right]+\left[b_{1}+\right], \ldots \ldots,\left[a_{r}^{+}\right]+\left[b_{r}^{+}\right]\right.}\left(Q_{1}, \ldots \ldots, Q_{r}\right)\right)_{H^{+} \times H^{+}}$

$$
\begin{aligned}
& =\operatorname{det}\left(\vartheta_{\left[a_{1}+\right]+\left[b_{1}+\right]}\left(Q_{1}\right) \vartheta_{\left[-a_{1^{+}}\right]+\left[b_{1^{+}}\right]}\left(Q_{1}\right)\right)_{\left\{[0],\left[\frac{1}{3}\right]\right\} \times\left\{[0],\left[\frac{1}{3}\right]\right\}} \cdots \cdots \\
& \quad \operatorname{det}\left(\vartheta_{\left[a_{r}+\right]+\left[b_{r^{+}}\right]}\left(Q_{r}\right) \vartheta_{\left[-a_{r^{+}}\right]+\left[b_{r^{+}}\right]}\left(Q^{r}\right)\right)_{\left\{[0],\left[\frac{1}{3}\right]\right\} \times\left\{[0],\left[\frac{1}{3}\right]\right\}} \not \equiv 0 \bmod p
\end{aligned}
$$

Since $\vartheta_{[a]}\left(Q_{1}, \ldots \ldots, Q_{r} \mid U_{1}, \ldots \ldots, U_{r}\right)$ is the specialization of $\vartheta_{[a]}(U \mid Q)$ over the replacement $Q_{i j}^{a}=1(i \neq j ; a \in Q)$. Hence we may conclude that for any prime $\mathfrak{p}$ it follows

$$
\operatorname{det}\left(\vartheta_{\left[a^{\prime}+b^{\prime}\right]}^{3}(Q)\right)_{H \times H^{\prime}} \not \equiv
$$




$$
\operatorname{det}\left(\vartheta_{\left[a^{+}\right]+\left[b^{+}\right]}(Q)_{\left[-a^{+}\right]+\left[b^{+}\right]}(Q)\right)_{H^{+} \times H^{+}} \not \equiv 0 \quad \bmod p
$$

Therefore we may put

$$
\begin{gathered}
\left(\gamma_{\left[a^{\prime}\right],\left[b^{\prime}\right]}(Q)\right)_{H \times H}=\left(\vartheta_{\left[a^{\prime}+b^{\prime}\right]}(Q) \vartheta_{\left[-a^{\prime}+b^{\prime}\right]}(Q) \vartheta_{\left[b^{\prime}\right]}(Q)\right)_{H \times H}\left(\vartheta_{\left[a^{\prime}+b^{\prime}\right]}^{3}(Q)\right)_{H \times H}^{-1} \\
\left(\beta_{[a],\left[c^{+}\right]}(Q)\right)_{G \times H^{+}}=\left(\vartheta_{[a]+\left[c^{+}\right]}(Q) \vartheta_{-[a]+\left[c^{+}\right]}(Q)\right)_{G \times H}\left(\vartheta_{\left[a^{+}\right]+\left[b^{+}\right]}(Q) \vartheta_{=\left[a^{+}\right]+\left[b^{\prime}\right]}(Q)\right)_{H^{+} \times H^{+}}^{-1}
\end{gathered}
$$

Then $\gamma_{\left[a^{\prime}\right],\left[c^{\prime}\right]}\left(Q_{1}, \ldots \ldots, Q_{r}\right)$ and $\beta_{[a],\left[c^{+}\right]}\left(Q_{1}, \ldots \ldots, Q_{r}\right)$ are the specializations of $\gamma_{\left[a^{\prime}\right],\left[c^{\prime}\right]}(Q)$ and $\beta_{[a],\left[c^{+}\right]}(Q)$ over the replacement $Q_{i j}^{a}=1(i \neq j ; a \in Q)$, respectively. From Lemma 3 for any prime $p$ it follows

$$
\begin{aligned}
\operatorname{det} & \left(\beta_{[a]+\left[c^{+}\right],\left[d^{+}\right]}\left(Q_{1}, \ldots \ldots, Q_{r}\right)\right)_{H^{+} \times H^{+}} \\
& =\operatorname{det}\left(\beta_{\left[a_{1}\right]+\left[c^{+}\right],\left[d^{+}\right]}\left(Q_{1}\right)\right)_{\left\{[0],\left[\frac{1}{3}\right]\right\} \times\left\{[0],\left[\frac{1}{3}\right]\right\}} \ldots . . \operatorname{det}\left(\beta_{\left[a_{r}\right]+\left[c^{+}\right],\left[d^{+}\right]}\left(Q_{r}\right)\right)_{\left\{[0],\left[\frac{1}{3}\right]\right\} \times\left\{[0],\left[\frac{1}{3}\right]\right\}} \\
& \neq \equiv 0 \bmod p
\end{aligned}
$$

This means that

$$
\operatorname{det}\left(\beta_{[a]+\left[c^{+}\right],\left[d^{+}\right]}(Q)\right)_{H^{+} \times H^{+}} \not \equiv 0 \quad \bmod p
$$

and proves Lemma 1 in $\S 1$

3.3 Finaly we shall give the proof of the main theorem:

Theorem 3. (The main Theorem). Let $G$ be a finite subgroup of odd order in $Q^{r} \mid \boldsymbol{Z}^{r}$ such that $G \supset H\left(=\frac{1}{3} Z^{r} \mid \boldsymbol{Z}^{r}\right)$. Let $K$ be a field containing $\gamma_{\left[a^{\prime}\right],\left[c^{\prime}\right]}(Q), \beta_{[a],\left[c^{+}\right]} Q$ $\left([\boldsymbol{a}],\left[\boldsymbol{c}^{\prime}\right] \in H ;[\boldsymbol{\alpha}] \in G ;\left[\boldsymbol{c}^{+}\right] \in H^{+}\right)$and $\mathfrak{q}$ be the ideal in the polynomial algebra $K\left[\left\{X_{[a]} \mid[\boldsymbol{a}] \in G\right\}\right]$ generated by the homogeneous elements:

$$
\begin{aligned}
& X_{\left[a^{\prime}\right]+[b]} X_{-\left[\boldsymbol{a}^{\prime}\right]+[b]} X_{[b]}-\sum_{\left[\boldsymbol{c}^{\prime}\right] \in H^{\prime}} \gamma_{\left[\boldsymbol{a}^{\prime}\right],\left[c^{\prime}\right]}(Q) X_{\left[\boldsymbol{c}^{\prime}\right]+[b]}^{\boldsymbol{3}}, \\
& X_{[a]+[b]} X_{-[a]+[b]}-\sum_{\left[c^{+}\right] \in H} \beta_{[a],\left[c^{+}\right]}(Q) X_{\left[\boldsymbol{c}^{+}\right]+[b]} X_{-\left[c^{+}\right]+[b]} \\
& \\
& ([\boldsymbol{a}],[\boldsymbol{b}] \in G ;[\boldsymbol{a}] \in H) .
\end{aligned}
$$

Then the map: $X_{[\boldsymbol{a}]} \rightarrow \vartheta_{[\boldsymbol{a}]}(Q \mid U)([\boldsymbol{a}] \in G)$ induces an isomorphism of $K\left[\left\{X_{[\boldsymbol{a}]} \mid[\boldsymbol{a}] \in G\right\}\right] / \boldsymbol{q}$ and $K\left[\left\{\vartheta_{[\boldsymbol{a}]}(Q \mid U) \mid[\boldsymbol{a}] \in G\right\}\right]$.

proof. Step $1^{\circ}$. Let $\mathfrak{p}$ be the kernel of the morphism:

$$
X_{[a]} \rightarrow \vartheta_{[a]}(Q \mid U)([a] \in G) .
$$


Since

$$
\operatorname{det}\left(\vartheta_{\left[a^{\prime}+b^{\prime}\right]}(Q)^{3}\right)_{H \times H} \neq 0, \quad \operatorname{det}\left(\vartheta_{\left[a^{+}+b^{+}\right]}(Q) \vartheta_{\left[-a^{+}+b^{+}\right]}(Q)\right)_{H^{+} \times H^{+}} \neq 0
$$

and

$$
\vartheta_{[a+b]}(Q \mid U)=Q(\boldsymbol{b}, \boldsymbol{b}) U(\boldsymbol{b})^{2} \vartheta_{[a]}(Q \mid Q(\boldsymbol{b}) U)
$$

it follows that $\left\{\vartheta_{\left[\boldsymbol{a}^{\prime}\right]}(Q \mid U)^{3} \mid[\boldsymbol{\alpha}] \in H\right\} \quad$ and $\quad\left\{\vartheta_{\left[\boldsymbol{a}^{+}\right]}(Q U)\left|\vartheta_{-\left[\boldsymbol{a}^{+}\right]}(Q \mid U)\right|\left[\boldsymbol{a}^{+}\right] \in H^{+}\right\} \quad$ are the basis of the modules of theta functions of type $(3 ;[0])$ and $(2 ;[0])$, respectively. Hence there exist $\lambda_{\left[a^{\prime}\right],\left[c^{\prime}\right]}$ and $\mu_{[a],\left[c^{+}\right]}$such that

$$
\begin{gathered}
\vartheta_{\left[a^{\prime}\right]+[b]}(Q \mid U) \vartheta_{-\left[a^{\prime}\right]+[b]}(Q \mid U) \vartheta_{[b]}(Q \mid U)-\sum_{\left[c^{\prime}\right] \in H} \lambda_{\left[a^{\prime}\right],\left[c^{\prime}\right]} \vartheta_{\left[c^{\prime}\right]+[b]}(Q \mid U)^{3}=0, \\
\vartheta_{[a]+[b]}(Q \mid U) \vartheta_{-[a]+[b]}(Q \mid U)-\sum_{\left[c^{+}\right] \in H^{+}} \mu_{[a],\left[c^{+}\right]^{+}} \vartheta_{\left[c^{+}\right]+\left[b^{+}\right]}(Q \mid U) \vartheta_{-\left[c^{+}\right]+[b]}(Q \mid U)=0
\end{gathered}
$$

Putting $U=Q\left(\boldsymbol{c}^{\prime}\right)$ or $U=Q\left(\boldsymbol{c}^{+}\right)\left(\left[c^{\prime}\right] \in H ;\left[\boldsymbol{c}^{+}\right] \in H^{+}\right)$, we have

$$
\begin{aligned}
& \lambda_{\left[\boldsymbol{a}^{\prime}\right],\left[\boldsymbol{c}^{\prime}\right]}=\gamma_{\left[\boldsymbol{a}^{\prime}\right],\left[\boldsymbol{c}^{\prime}\right]}(Q), \\
& \mu_{[\boldsymbol{a}],\left[\boldsymbol{c}^{+}\right]}=\beta_{[a],\left[\boldsymbol{c}^{+}\right]}(Q)\left(\left[\boldsymbol{a}^{\prime}\right],\left[\boldsymbol{c}^{\prime}\right] \in H ;[\boldsymbol{a}] \in G ;\left[\boldsymbol{c}^{+}\right] \in H^{+}\right) .
\end{aligned}
$$

This proves $\mathfrak{p} \supset \mathfrak{q}$.

Step $2^{\circ}$. Let $k$ be a subfield in $K$ such that $\gamma_{\left[a^{\prime}\right],\left[c^{\prime}\right]}(Q), \beta_{[a],\left[c^{+}\right]}(Q)\left(\left[\boldsymbol{a}^{\prime}\right],\left[\boldsymbol{c}^{\prime}\right] \in\right.$ $\left.H ;[\boldsymbol{a}] \in G ;\left[\boldsymbol{c}^{+}\right] \in H^{+}\right)$are transcendental over $k$ and put $K^{\prime}=k\left(\left\{\gamma_{\left[\boldsymbol{a}^{\prime}\right],[\boldsymbol{c}]}(Q)\right.\right.$, $\left.\left.\beta_{[a],\left[c^{+}\right]}(Q)\right\}\right)$ and $K^{\prime \prime}=k\left(\left\{\gamma_{\left[a^{\prime}\right],\left[c^{\prime}\right]}\left(Q_{1}, \ldots \ldots, Q_{r}\right), \beta_{[a],\left[c^{+}\right]}\left(Q_{1}, \ldots \ldots, Q_{r}\right)\right\}\right)$. We denote by $\mathfrak{q}^{\prime}$ and $\mathfrak{p}^{\prime}$ the ideals in $K^{\prime}\left[\left\{X_{[\boldsymbol{a}]} \mid[\boldsymbol{a}] \in G\right\}\right]$ such that $\mathfrak{q}^{\prime}=\mathfrak{q} \cap K^{\prime}\left[\left\{X_{[\boldsymbol{a}]} \mid[\boldsymbol{a}] \in G\right\}\right]$ and $\mathfrak{p}^{\prime}=\mathfrak{p} \cap K^{\prime \prime}\left[\left\{X_{[a]} \mid[\boldsymbol{a}] \in G\right\}\right]$. We mean by $\mathfrak{q}^{\prime \prime}$ and $\mathfrak{p}^{\prime \prime}$ the ideals in $K^{\prime \prime}\left[\left\{X_{[a]} \mid\right.\right.$ $[\boldsymbol{a}] \in G\}]$ which are the specializations of $\mathfrak{q}^{\prime}$ and $\mathfrak{p}^{\prime}$ over the replacement $Q_{i j}^{a}=1$ $(i \neq j, a \in \boldsymbol{Q})$. Then by virtue of Theorem 2 we have $\mathfrak{p}^{\prime \prime}=\mathfrak{q}^{\prime \prime}$. Let $\mathfrak{q}_{n}^{\prime}$ and $\mathfrak{p}_{n}^{\prime}$ be the $K^{\prime}$-submodules in $\mathfrak{q}^{\prime}$ and $\mathfrak{p}^{\prime}$ consisting of all the elements of degree $n$, respectively. Then it is sufficient to prove $\mathfrak{p}_{n}^{\prime}=\mathfrak{q}_{n}^{\prime}$. Let us denote by $\mathfrak{q}_{n}^{\prime \prime}\left(=\mathfrak{p}_{n}^{\prime \prime}\right)$ the $K^{\prime \prime}$-submodule of $\mathfrak{q}^{\prime \prime}\left(=\mathfrak{p}^{\prime \prime}\right)$ consisting of all the elements of degree $n$.

Since $\mathfrak{q}^{\prime \prime}$ is the specialigation of $\mathfrak{q}^{\prime}$, it follows

$$
\operatorname{dim}_{K^{\prime}} \mathfrak{q}_{n}^{\prime} \geq \operatorname{dim}_{K^{\prime \prime}} \mathfrak{q}_{n}^{\prime \prime}=\operatorname{dim}_{K^{\prime \prime}} \mathfrak{p}_{n}^{\prime \prime}
$$

If we denote by $g$ the order of $G$, then from Proposition 1 , Theorem 2 and Step $1^{0}$ we have 


$$
\begin{aligned}
g n^{r}= & \left(\begin{array}{c}
g+n-1 \\
n
\end{array}\right)-\operatorname{dim}_{K^{\prime \prime}} \mathfrak{p}_{n}^{\prime \prime}=\left(\begin{array}{c}
g+n-1 \\
n
\end{array}\right)-\operatorname{dim}_{K^{\prime \prime}} \mathfrak{q}_{n}^{\prime \prime} \\
& \geq\left(\begin{array}{c}
g+n-1 \\
n
\end{array}\right)-\operatorname{dim}_{K^{\prime}} \mathfrak{q}_{n}^{\prime} \geq\left(\begin{array}{c}
g+n-1 \\
n
\end{array}\right)-\operatorname{dim}_{K^{\prime}} \mathfrak{p}_{n}^{\prime}=g n^{r}
\end{aligned}
$$

This means that $\mathfrak{p}_{n}^{\prime}=\mathfrak{q}_{n}^{\prime}(n=1,2, \ldots \ldots)$ and thus $\mathfrak{p}^{\prime}=\mathfrak{q}^{\prime}$. This completes the proof of Theorem.

\section{ReFERENCE}

[1] H. Morikawa: On the Explicite Defining Relations of Abelian Schemes of Level Three p. 143-157 Nagoya Math. Jour. Vol. 27-1 (1966).

Institute of Mathematics Nagoya University 